\title{
Self-repairing material systems-a dream or a reality?
}

\author{
Hartmut Fischer ${ }^{1,2}$ \\ ${ }^{1}$ TNO Science and Industry, Eindhoven, The Netherlands; \\ ${ }^{2}$ CSIRO Material Science and Engineering, Clayton South, Australia; hartmut.fischer@tno.nl
}

Received 21 April 2010; revised 22 June 2010; accepted 27 June 2010.

\begin{abstract}
Currently, most industrial materials rely entirely on passive protection mechanisms; such mechanisms are readily applicable and universal for many different materials systems. However, they will always stay passive, and therefore their lifetime and functionality is limited and related to the amount of protective additives and the intensity of their consumption. Therefore, better, and preferentially active process for the protection/repair of damaged materials-self-repairingprocesses-were developed and need to be developed further. Although it sounds futuristic or like a fiction in the modern, trendy times, which in many ways affects also directions of research; self healing of material systems exists already for a long time in all sorts of systems of materials or functionalities. The aim of this work is to go beyond the scope of a classical review the ones published recently in this field which almost entirely focused only onto polymeric systems. In this work, an analysis of the underlying functional and constructional principles of existing natural and synthetically self-healing systems spanning over a range of classes of materials is given leading to general rules and principles for the design of new and application tailored self-healing material systems.
\end{abstract}

Keywords: Self Healing; Systems; Sensors

\section{INTRODUCTION}

Self-healing is an intrinsic property of living organisms, enabling them to cope with all sorts of damage or injury they experience during their lifetime. This repair occurs with essentially no external intervention. Thus, wounds heal, broken bones heal, and even lost parts of living bodies (lizard tails etc.) can be replaced in some cases. Some natural self-healing composite systems such as bones go beyond simple healing to the extent that they remodel themselves continuously. Damaged material is removed and replaced by new material and over-designed material is also removed and structures under stress are enhanced by additional material. To enable this process, specific cells entrapped in the bone tissues act as strain sensors and feel large deformations. Subsequently, signals are sent to cells responsible for removing or forming material (bone) [1].

In order to deal with the phenomenon and the connected principles of self-healing in general, and even further to design and construct artificial self-healing functionalities for systems and devices, it is important to understand the nature and the consequences of damage and of degradation processes first.

Damage in general terms can be described as changes introduced to a system that affect its function and/or performance. That means that damage is not meaningful without a comparison of two different states of the system in question, one of which is assumed to be the initial (and often un-damaged) state. The term damage does not necessarily imply a total loss of system functionality, but rather a departure in level of system operation from optimal. A fundamental challenge is the fact, that damage is typically a local phenomenon and may be therefore difficult to detect (in time) [2].

Most materials (systems) lose their integrity, their value, operation or usefulness over time due to degradation processes where defects grow and coalesce to cause component and finally system level damage (e.g. fatigue or corrosion damage accumulation). On a relatively short time scale, damage can also result from scheduled discrete events such as aircraft landing and from unscheduled discrete events such as an impact. As damage grows, it will reach a point where it affects the system operation to a level, which is not any longer acceptable for a user; this point is referred to failure.

The main causes of deterioration of materials may be summarised as:

1) Harmful materials, substances and agents such as oxygen, oxidizing agents, water, salts, poisons, active materials, and living bodies such as virus, bacteria, fungi, 
insects, animals, human beings and others,

2) Factors from the surroundings such as heat, visible light, external mechanical force e.g. strong wind, radiation, pressure, rain, collapse of the adjacent structure, a sudden impact and others.

Such deterioration (or damage) are in general irreversible, they occur progressively and exponentially in time until a certain threshold is reached where either significantly high costs for repairs are necessary or a (fatal) failure is going to occur. Increased lifetime and reliability of materials systems and devices can be critical requirements in traffic, construction, information transfer, medicine, military, space missions as well as in ordinary daily life.

In order to prevent such detoriation the following response mechanisms are known:

PASsIVE (Built-in damage prevention): by protection agents, that are directly attacked by the deterioration and the concentration of the agent is directly related to the amount of protection and that provide no repairability and hence no memory of the inherent structure.

ACTIVE (Autonomous or self-repair): here the deterioration attacks directly atoms and molecules of the material itself instead of protection agents. The amount of repairing agents needed depends on the concentration of damaged sites and the accumulation of damage in time and at certain locations as well as on the repair rate; a transfer of material is needed and a memory of the original structure is preferable.
The pictures in Figure 1 show schematically the described mechanisms applied to the case of polymers matrices as damaged material.

Currently, most industrial materials rely entirely on passive protection mechanisms; such mechanisms are readily applicable and universal for many different materials systems. However, they will always stay passive, and therefore their lifetime and functionality is limited and related to the amount of protective additives and the intensity of their consumption.

Therefore, better, and preferentially active processes for the protection/repair of damaged materials-selfrepairing-processes-were developed and need to be developed further. Although it sounds futuristic or like a fiction in the modern, trendy times, which in many ways affects also directions of research; self healing of material systems exists already for a long time in all sorts of systems of materials or functionalities.

The aim of this work is to go beyond the scope of a classical review which almost entirely focused only onto polymeric systems like the ones published recently in this field [4-6]. In this work, an analysis of the underlying functional and constructional principles of existing natural and synthetically self-healing systems spanning over a range of classes of materials is given leading to general rules and principles for the design of new and application tailored self-healing material systems. Therefore, specific choices from the existing literature

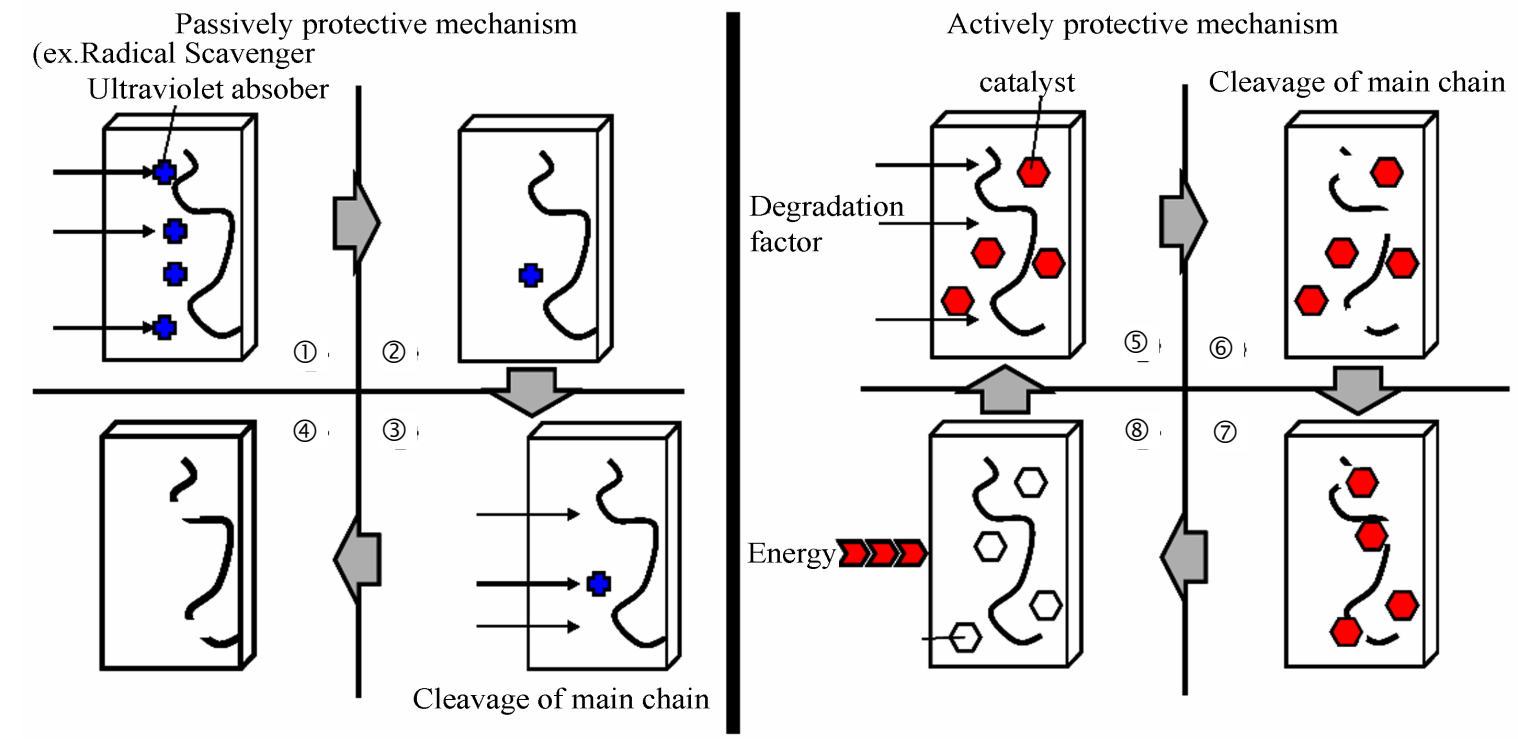

Figure 1. Passively protective mechanism versus active protective mechanism. Reference [3]: 1) Degradation factors are prevented from interacting with polymer chains, 2) Concentration of stabilizer deteriorates, 3) Degradation factors interact directly with polymer chains, 4) Degradation of polymeric chains, 5) Degradation factors directly interact with polymer chains and degradation occurs temporarily, 6) Cleavage of main chain, 7) Catalytic re-bonding reaction occurs, 8) Catalyst becomes reactivated. 
have been made for this analysis, which either show new principles or contribute to this aim without repeating already existing systems in a similar or slightly different way.

In the next section, a discussion of the phenomenon of repair and especially self-repair is presented, together with the principal design features and requirements that enable a self-repair to take place.

Examples of self-repairing systems in material and life science starting with assisted (stimulated) repairing and of self repairing systems already known or currently under development are discussed in Section 3. This is followed by a discussion of the sensors and triggers employed or potentially employable in self-healing systems in Sections 4 and 5. Finally, conclusions are drawn with respect to requirements for the design of self-healing systems and working areas necessary to concentrate on are presented (in the Sections 6 and 7).

\section{WHAT IS SELF REPAIR?}

Repair itself is a process which can be initiated after damage on a local or global scale of a given functional system with the aim, to reduce the local or global level of damage and to extend or to renew the functionality and life-time of the damaged part, system or device.

Self-repair is then in principle an autonomically initiated response to damage or failure. In order to perform this repair, any self-repair system must be capable of 1) identifying and 2) repairing failures. If failure is classified as 'any occurrence, which results in the system deviating from its original task or not being any more able to fulfil its function', what is than ultimately classified as 'repair'? (Self) repair can be classified under two significant approaches:

Attributive repair and

Functional repair

Attributive repair are attempts to restore the attributes of the system to their original state, the full capacity of the system.

Functional repair are attempts to restore the function of the system. If full functionality cannot be restored, this strategy attempts to focus the remaining available resources to maximise the available functionality.

Attributive repair is the optimal solution. If the attempt to restore the system to its complete original condition fails, there is still significant benefit to be gained in most instances if the system continues to operate, even with reduced functionality.

Attributive self-repair is an intrinsic property of nature. Figure 2 shows a schematic example of the steps in a self-repair cycle in nature, in this case bone failure due to fracture. This autonomic healing is initiated by the

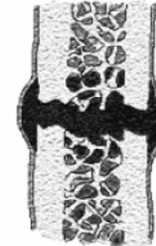

(a)

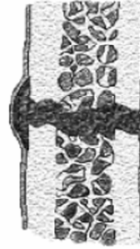

(b)

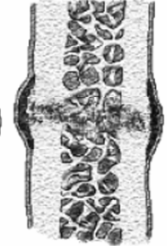

(c)

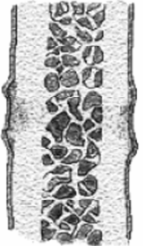

(d)

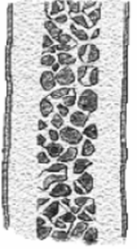

(e)
Figure 2. Schematic picture of the healing stages of bone: a) internal bleeding (sensor), forming of a fibroin cloth; b) unorganized fiber mesh develops (first step of (primitive) repair); c) calcinations of the fibrocartilage (second step of repair); d) calcification converted into fibrous bone (structural repair); e) transformation into lamellar bone (complete recovery of intrinsic structure and functionality) [7].

local damage of the vascular blood system. Healing a bone requires energy in the form of nutrition together with the delivery of cell material to the fracture site by the network of blood vessels; for healing a sufficient healing time must elapse. The healing process consists of multiple stages of deposition and assembly of material.

Mimicking nature is the ultimate goal for materials designers and developers.

There are essentially two design approaches to realize self-repairing systems:

1) a conventional design modifying existent design philosophies by incorporating intelligent techniques at the design stage to obtain a system with sufficient autonomous capabilities to initiate and effect a functional and in ideal cases an attributive repair.

2) a radically new design philosophy, where the system is designed in a cellular structure such that each cell contains a (genetic) code, which defines and determines its functionality. This term is called embryonics and it attempts to replicate the cellular structure of organisms in nature and their inherent self-replicating and thus self-healing capabilities resulting mostly in attributive repair.

Although both strategies will certainly lead to new self-repairing systems, the conventional process will be easier to implement in the short term.

Generally, self-repair processes in smart systems should not only replace any passive protection mechanism but should finally lead to structures, which are able to sense its internal state, to monitor the healthiness of the material/functionality/system/device at any time and at any location and respond in a manner that fulfils its functional requirements based on the gained information-sensing/detection, diagnosis of failure and activating of repair!

In order to perform properly, a smart system must have incorporated (embedded or surface mounted) ac- 
tuation and sensor elements which offer the capacity of health monitoring (to be able to diagnose, interpret and correct structural faults in situ as they occur) by measurement of physical quantities such as vibration, permeability, current flow, strain, acoustic emission, impedance, $\mathrm{pH}$-changes, colour tracers, etc., that are informative with respect to the state of structural health. Methods and instruments are X-ray tomography, ultrasonic methods and eddy current methods and strain- gauges, optical fibres, piezos and in general "smart tagged" composites.

Specifically, information relating to the severity, significance and location of damage is required. Therefore, a detection of:

1) Damage occurrence,

2) Damage location,

3) Damage type

4) Damage force magnitude and consequently

5) The remaining lifetime of the structure is necessary for maintaining the functionality of the material/structure/device. The information obtained has to be fed into the recovery cycle as sketched in Figure 3.

The interpretation and operational evaluation of the obtained data may include on top of the data acquisition a normalisation and filtering process, a feature selection and information condensation and finally a statistical model development for feature discrimination. This diagnosis will lead to an eventual initiation of the repair action.

Finally, to perform a repair action, mobile parts are needed to be transported to the location of the damage. This can be realised using a liquid either locally available or available via a vascular network system like the blood system in living organisms but also a transport via the gas phase is possible, in case material for the repair action is needed.

Self-healing or self-repair can therefore in summary be defined as "the ability to substantially return to an initial, proper operating state or condition prior exposure to a dynamic environment by making the necessary adjustments to restore to normality and/or the ability to resist the formation of irregularities and/or defects". It is a much more effective damage management decreasing damage level during certain stages of life time than passive prevention, since it is only applied in cases, times and locations of real need-damage which jeopardizes the functionality of a given system. Ideally, a self-healing system will therefore display an unlimited lifetime since the rate of damage formation is negative or zero.

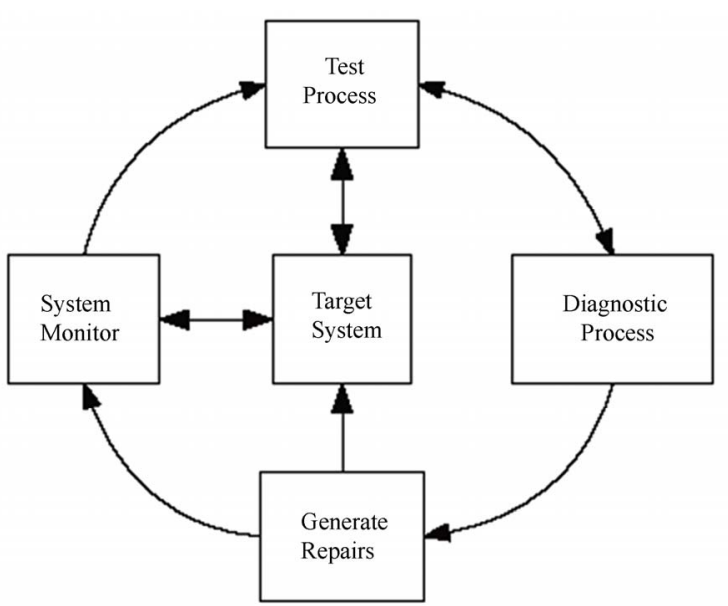

Figure 3. Health monitoring and repair cycle for an active smart self-repairing system [8]. The system monitor must be able to analyse system performance against predefined performance benchmarks in order to continuously verify correct operation of the process. The testing process also must have bi-directional communication to activate specific test sequences and receive results. This process should exploit any built -in self-test ability, and the testing results are forwarded to the diagnostic process. Automating the fault diagnosis process inherently demands the application of intelligent techniques. Finally, the self-repair process must be able to affect a repair based on the reduction in resources available, following isolation of the faulty components. If the original level of performance cannot be restored, the monitoring system must be modified to reflect the changes in system characteristics. This feature demands an optimisation capability. Reprinted from Engineering Applications of Artificial Intelligence, Self-repair of embedded systems, 17, E. A. Coyle, L. P. Maguire, T. M. McGinnity, 1-9, Copyright (2004), with permission from Elsevier.

\section{EXAMPLES OF SELF REPAIRING SYSTEMS IN MATERIAL AND LIFE SCIENCE}

In this section a number of existing self-repair systems is critically analysed with respect to its design, functional units/modules and their action/performance.

Within all classes of materials, the self-repair potential of polymers is probably the largest of all materials, since the polymeric nature offers more possibilities of developing and displaying molecular mobility than any other material. However, and as discussed below, this does not exclude other materials from a participation in functional self-healing systems.

The different options for the design of the repair action of a polymeric based system connected with examples of how this can be realized are illustrated in Figure 
4 [9] and will be discussed in the following paragraphs.

Essentially a differentiation between nature-analogous true autonomous self-repairing systems and other systems, which may be only able to react onto damage and to make the necessary adjustments to restore itself to normality with human intervention, and may be therefore better called assisted healing systems, can be made. Assisted (local) healing can be achieved, e.g. by an increase of temperature, by irradiation or by other stimuli.

\subsection{Thermally Stimulated Repair}

A repair of accumulated damage may be triggered by thermal stimulation where the system allows the flow of material, wetting of the crack surfaces and gluing them together in a heating-cooling cycle.

This can be most easily achieved in a system containing polymeric chains, which during the transition from the glassy to the rubbery state experience a huge increase in mobility and hence the possibility to re-entangle after physical separation.

The model to explain the movement of a polymer molecule in a snake-like fashion inside a cross-linked polymeric gel, also known as the reptation model as proposed by de Gennes [10] implies also a possibility of polymeric chains to repair damage. Prager and Tirell [11], Jud and Kausch [12] and later Wool and O'Connor [13] used the same reptation model to determine the time required for healing caused by the inter-diffusion of molecules between crack faces and thus re-gaining strength by annealing just above the $\mathrm{T}_{\mathrm{g}}$. Kim and Wool [14] predicted theoretically the recovery process as a function of healing time confirming existing experimentaldata [15]. Also, it has been shown that, if amorphous bulk samples of high-molecular-weight polystyrene (PS) were brought into contact with themselves in a lap-shear joint geometry below the bulk glass transition temperature, an increase in lap-shear strength and fracture occurred $[16,17]$. This can be attributed to the time-temperature shift of the glass transition, as well as to the possible enhanced surface molecular mobility of polymer chains leading to a lower $T_{g}$ at free polymer surfaces compared to the bulk $T_{g}$ which enables inter-diffusion of chains and self-healing even below $\mathrm{T}_{\mathrm{g}}$ ! At the present time, a decrease in density and a decrease in the entanglement density close to the polymer surface can be considered as the factors contributing to the increased molecular mobility at polymeric surfaces in comparison with that in the bulk [18]. In addition to these factors, a "jump" in the conformational entropy of the chain segments located at the surface (these segments have a decreased entropy imposed by the polymer-air interface [19] upon the contact of the surfaces) may be considered as one of the driving forces of inter-diffusion across the interface.

In principle, self-repairing polymers can therefore be realized essentially attributed to molecular inter-diffusion. Polymer networks with dangling chains can there-

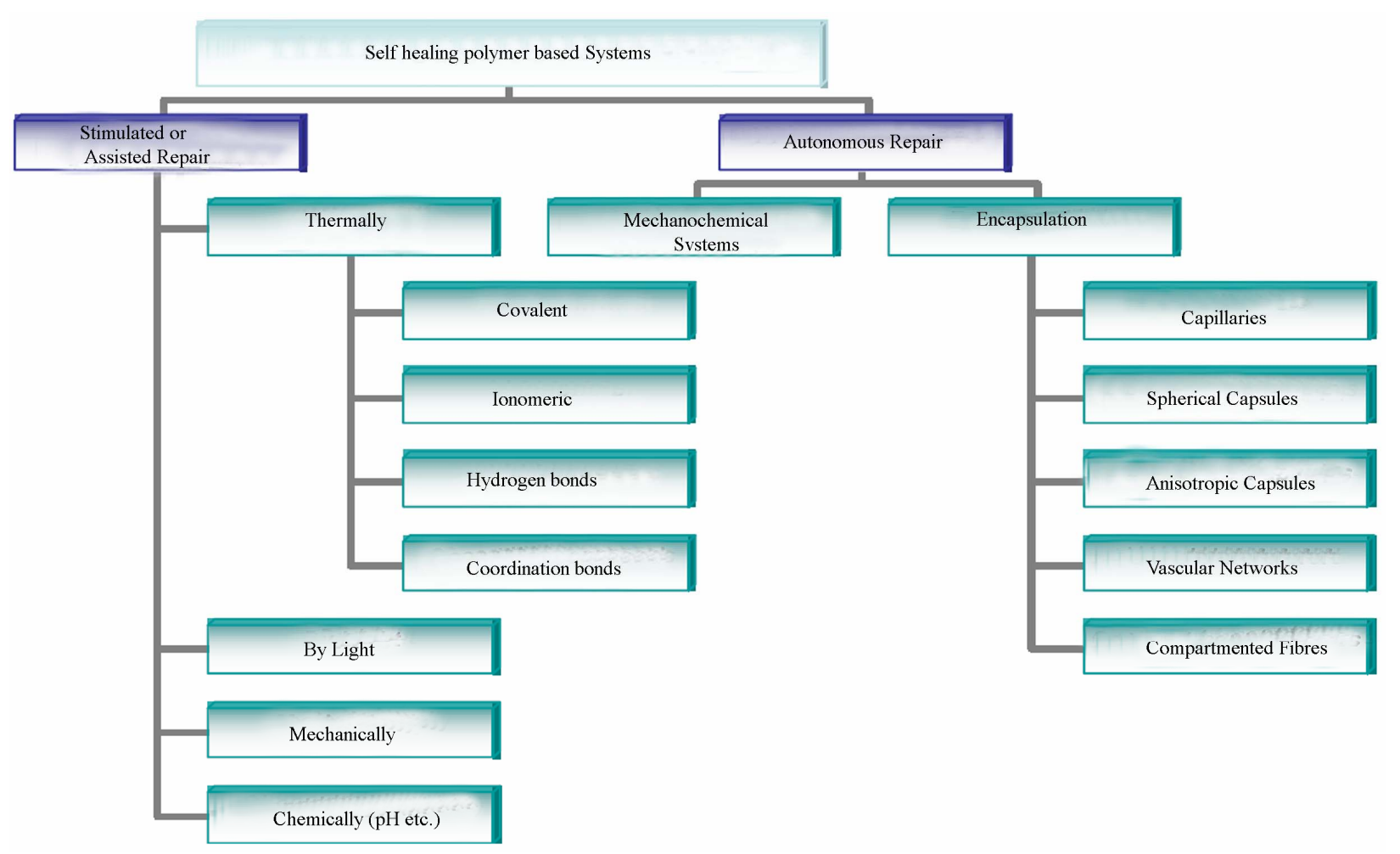

Figure 4. Organization of self-healing polymer based material systems according to the different principles employed adapted from [9]. 
fore be employed to heal cleavages at room temperature without any manual intervention [20].

However, self-repair needs time and rest. In a later study, the concept of re-entanglement of polymeric chains by reptation has been applied in thermoset composite materials where a linear thermoplastic polymer material was inserted into an epoxy matrix leading to a recovery of up $70 \%$ of the fracture toughness [21]. In this case as in the previous cases, multiple repairs are possible [22].

Alternatively, Zako and Tanako embedded small grainparticle adhesives in a glass epoxy composite laminate [23] an Meure, Wu and Furman polyethylene-co-methacrylic acid particles in an epoxy resin [24] to act as repair agents. The 50 micron sized particles consisted of thermosetting type epoxy; the matrix was a cold-setting epoxy. Damage can be repaired by the particles acting as repairing actuator when melted by heat. A similar mechanism can be used for the thermally stimulated self-repair of glass-ceramic composites, here heals the flow of the viscoelastic glass matrix at higher temperatures aging induced micro-voids in the composite material [25].

A very efficient and fast assisted repairing system by external stimulation consist just of one material capable of fulfilling all functions (structural properties, sensor and providing of mobility) by reversible formation of covalent or non-covalent bonds (ionic or H-bonds). Thermally controlled covalent bond formation is already long known in organic chemistry and may be applied to the formation of linear polymers as well as to the formation of polymer networks. Besides thermally reversible urea [26], alkoxyamine units incorporated in the main chain [27], nitroso-dimerisation and ester formation under presence of cyclic anhydrides [28], the $(4+2) \mathrm{Di}-$ els-Alder reaction is by far the most important reaction. It was already discovered in 1928 [29] and honoured with the Nobel Prize in 1950 and generally considered the "Mona Lisa" of reactions in organic chemistry since it requires very little energy for the formation of a covalently connected ring-structure. This reaction enables step polymerizations and cross-linking reactions together with a thermally reversibility and is therefore ideally suited for thermally stimulated polymer repair and potentially, recycling. Mostly, but not exclusively, the reaction between furane and maleimide derivatives is used for the generation of a thermally reversible bond. This has been applied already in 1969 for the synthesis of reversibly cross-linked networks, which yielded a tough rubbery film at $100^{\circ} \mathrm{C}$ and returned to a re-mouldable polymer at $140^{\circ} \mathrm{C}$ [30]. Subsequently, this principle has been further developed and the suitability of the system for self-healing bulk materials demonstrated [31-33]. These thermally reversible bond (cross-link) formations leading to self-healing properties upon thermal stimulation using the Diels-Alder reaction (see Scheme 1) can be used in application areas like coating systems [34].

Using this concept, powder coatings can be applied in their cross-linked state, which has distinct advantages with respect to their storage stability. When heated above the threshold temperature flow should be sufficient to enable proper film formation. As soon as the surface is of sufficient quality, cross-linking can be started-simply by cooling down! In a fully cross-linked state of a powder coating system, i.e., below a certain threshold temperature, the coating will exhibit its essential properties with respect to mechanical strength, etc., but in its (partly) de-cross-linked state, above the threshold temperature, it will show a certain level of plasticity and flow. This plasticity will decrease problems in the area of film formation of powder coatings, will enable repair of the coating using their self-healing properties (see Figure 5).
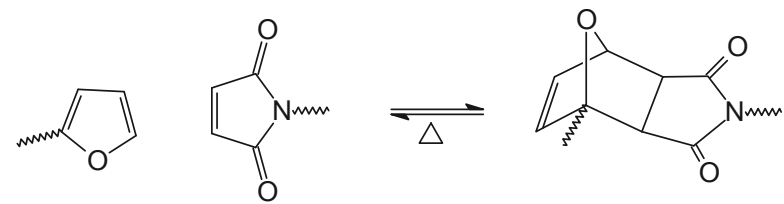

Scheme 1. Schematic representation of the Diels-Alder reaction used for thermally stimulated repair.

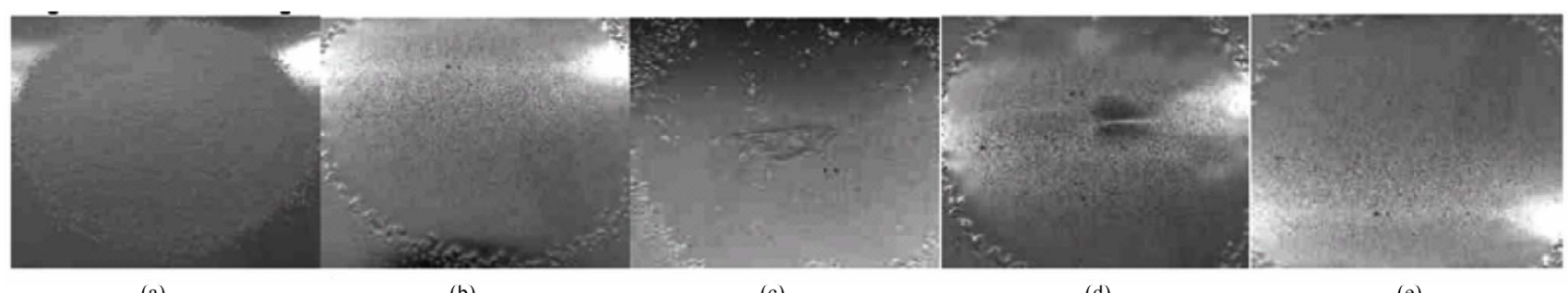

(a)

(b)

(c)

(d)

(e)

Figure 5. Visualisation of the self-healing of a cross-linked powder coating based on the acrylate copolymers: a) crosslinked powder, b) molten powder, c) damaged coating, d) re-flow, e) repaired coating [34], scale bar $=5 \mathrm{~mm}$. 
Besides application in coatings [35-38], reversibly cross-linkable materials are of interest for several areas of applications including thermally reversible bonding [39], thermally reversible solubility of polymers [40], processes connected with the recycling of plastics [37], encapsulates which open at elevated temperatures, reversible data storage medium [41] and also as matrix material in composites $[42,43]$. Here, polymeric materials with dicyclopentadiene units in the polymer backbone were used as a matrix for re-mending composites reinforced with graphite fibers [42]. The graphite fibers are used as electrical conductors to provide the necessary heat to the polymer. Microcracks, introduced by bending the substrate were healed by applying electric currents. Alternatively, arrays of conductive, electromagnetic scattering elements such as copper wires and coils were incorporated together with reinforcing fibres into a matrix consisting of a thermally reversible crosslinked networked base on a retro-Diels-Alder mechanism [43]. Since the wires are uniformly distributed within the matrix they can be used to heat the composite quick and uniformly by absorption of electromagnetic radiation. Furthermore, the reinforcing fibres are also contributing to the healing mechanisms, since they display a negative coefficient of thermal expansion (Kevlar: -2 to $-6 \mathrm{ppm} / \mathrm{K}$ ) and ensure therefore a mechanical closure of cracks while raising the temperature and increasing the healing efficiency in such a way to a great deal.

In a similar way, embedded shape-memory alloy wires [44] as well as a syntactic shape memory polystyrene foam [45] have been used to improve the performance of an existing self healing system. The improvement in performance due to crack closure enables multiple selfhealing actions and increases crack fill factors during the repair action.

Non-covalently bonded polymeric systems are also known to heal upon thermal treatment. For example a supramolecular network can be established by thermally reversible $\mathrm{H}$-bonds leading to rearrangements of the molecules in the non-bonded, heated state and reformation of the network in the "cold" state $[46,47]$.

A reversible formation of ionomeric "cross-links"clusters, which are formed by phase separation of the charged parts of macromolecules from the neutral chain segments can be used also as a self-repair mechanism being repeatable many times depending primarily on the stability of the polymer. Although it is not an autonomic process, it is activated when thermal energy is transferred to the polymer. During high-speed impact, energy is absorbed which is elastically stored and dissipated as heat. This increases the local temperature of the impacted polymer above the melting point (disrupting the physical cross-links) while having little effect upon the temperature of the surrounding matrix. The ionic domains persist in the melt so that the polymer can be elongated to high levels of strain and rebound elastically when the stored energy is released at failure. The level of stretching enables the polymer to seal the cavity as it returns to its original position, but it is the viscoelastic properties and the capacity of physical cross-links to reform that determines the final level and strength of healing. The structural integrity of the polymer provides sufficient strength in the melt to prevent other deleterious effects from polymer flow $[48,49]$.

\subsection{Non-Thermal Stimulation of Self Repair}

In a similar manner to the retro-Diels-Alder compounds, reversible cross-links can be established by photo induced cross-linking, which can be reversed by irradiation with a different wavelength to heal cracks, such as with cinnamic acid derivatives [50].

A covalently cross-linked network containing ally sulfides is able to undergo photomediated, reversible cleavage of its backbone to allow chain rearrangement for rapid stress relief at ambient conditions without mechanical property degradation [51]. The key to this reversible backbone cleavage is addition-fragmentation chain transfer. A reaction diffusion of radicals through the cross-linked matrix occurs initially by reaction of a radical with an in-chain functionality, forming an intermediate, which in turn fragments, reforming the initial functionality and radical. This addition-fragmentation process alters the topology of the network, but the polymer chemistry and network connectivity remain unchanged.

Alternatively, also an electrical current run through a conductive material system may be used to assist healing [52]. By imparting conductive properties into these materials, one may obtain 'real-time' status of a material's structural integrity through electric feedback mechanisms. This feature could lead to new approaches for detecting and quantifying microcracks which further could lead to materials capable of recording their stress/load histories. Other possibilities include using electric fields or currents as healing function. Upon the formation of a microcrack, the total number of electron percolation pathways within the material should decrease. As a result, its inherent electrical resistance should increase accordingly. If the material is integrated into a circuit, the drop in conductivity could be used to trigger a simultaneous increase in the applied electric field. Considering that the microcrack is the source of the increased resistance, this voltage bias should result in the generation of heat localized at the microcrack. By harnessing the generated thermal energy to overcome kinetic barriers, the system may be electrically driven 
back to its original (i.e. a low resistance/high-current) state. This has been shown using N-heterocyclic carbines and transition metals [52].

It is well known that "crack healing" of polymers is observed via stimulation using a specific solvent. This phenomenon is called "solvent healing". In this healing process, the solvent is applied onto the fractured polymer until healing has occurred and then removed. The observed crack healing is a superposition of two different mechanisms: firstly, the formation of a close contact of the crack surfaces initiated by internal compression induced by surface swelling, and secondly, the self-diffusion of polymer chains across the interface forming the physical links (entanglements) between polymer chains coming from different sides of the interface promoted by the action of the solvent lowering locally the $T_{g}[53,54]$. This concept can also be used for a built-in self-healing system, as described below.

\subsection{Synthetically Designed Autonomously Self-Repairing Systems}

\subsubsection{Ceramic Systems}

An existing self-healing material or system can be found in the layers applied in barrier systems (liners) to ensure security of deposits of dangerous waste. Here, geo-synthetic clay liners (GCLs), which contain natural sodium bentonite encapsulated between geo-textile components, are the sealing elements [55]. Desiccation cracks, which cause a significant increase of the permeability, can be repaired by the self-sealing properties of calcium and sodium bentonite.

Alternatively, self-healing sealing elements based on the principle that two or more parent materials (for example pozzolanic material and lime containing material)

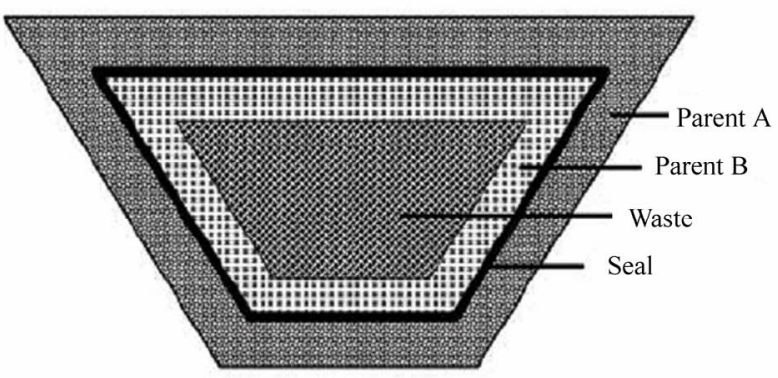

(a) placed in vertical or horizontal layers, are described. These materials will react at their interfaces to form insoluble, barrier reaction products and heal themselves after being fractured (see Figure 6 [56]).

Curiously, the same strategy has been operating in the construction for centuries. Many ancient Roman constructions made from limestone survived only because of the self-healing capacity of limestone in interaction with moisture/water. The phenomenon of self-healing in concrete has been known for many years [57-62]. Cracks in reinforced concrete are unavoidable and the corrosion of reinforcing steel due to de-icing salts or sea water is a major course of deterioration of reinforced concrete structures while disrupting the naturally formed corrosion protective passivation film. It has been observed that some cracks in old concrete structures are lined with white crystalline material suggesting the ability of concrete to self-seal the cracks with chemical products $\left(\mathrm{CaCO}_{3}\right)$, most likely with the aid of rainwater and carbon dioxide (see Figure 7). The healing of cement results in the voids being filled with hydration products which have a bulk volume approximately 2.1 times the original volume [63], an effect which is discussed below. However, efficient self-healing occurs only for cracks not exceeding a certain width (ca 135 microns) [64].

Unfortunately, this mechanism not always work in normal concrete because the width of the tensile cracks cannot be easily controlled or tuned. Localized fracture leads to continued increases in crack width under decreasing tensile load, and rapidly exhausts the amount of chemicals available for crack sealing and composite re-healing. Thus, it is critical that the tensile crack width is controlled and must be limited to a few tens of microns. Again, the success of the repair action depends

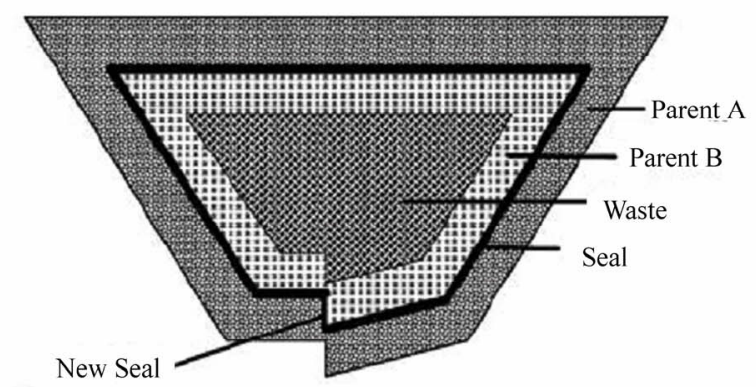

(b)

Figure 6. Schematic illustration of the self-sealing/self-healing feature of a pozzolanic and lime containing barrier material system: a) before fracture; b) after fracture. Reprinted from Waste Management, Laboratory development and field demonstration of self-sealing/self-healing landfill liner, 25, C. Shi, R. Booth, 231-238, Copyright (2005), with permission from Elsevier. 
strongly on the damage degree accumulated. When the damage degree is less than the threshold, the self-healing ratio of concrete is increased with the increase in damage degree; once the damage degree exceeds the threshold, the self-healing ratio is decreased with the increase in damage degree [65].

In recent years, fiber-reinforced cement-based composites optimized for ultra high tensile ductility with a minimized amount of fibres have been designed based on micromechanics design tools [66]. Such materials, defined as Engineered Cementitious Composites (ECC), display "plastically yield" under excessive loading through controlled micro-cracking while suppressing brittle fracture localization and thus enabling self-repair actions.

The potential of calcite-precipitating bacteria for concrete or limestone surface remediation or durability improvement has only recently been investigated and is a possible alternative to repair cracks in concrete [67].

In the case of composite materials, it is also possible to use an inorganic phase to perform the self-repairing process, as demonstrated in the case of decalcification-hydration reactions of hydraulic (CA) fillers in poly-phenylenesulfide (PPS) coatings used in geothermal applications. The hydraulic fillers, which can heal and repair micro-sized cracks appearing on the surfaces of corrosion protection coatings, are embedded into the matrix material (PPS) [68]. The decalcification-hydration reactions of the $\mathrm{CaO}-\mathrm{Al}_{2} \mathrm{O}_{3}$ and $\mathrm{CaO}-2 \mathrm{Al}_{2} \mathrm{O}_{3}$ reactants present and exposed in the cracks lead to the rapid growth of boehmite crystals, densely filling and sealing the cracks, while the calcite leaches out of cracks because of the formation of water-soluble calcium bicarbonate (see Figure 8).

Healing and repairing of micro-sized cracks generated on the surfaces of the PPS coating was observed after exposure of the cleaved coatings to a simulated geothermal environment $\left(200^{\circ} \mathrm{C}, \mathrm{CO}_{2}\right.$-loaded brine $)$. During exposure for $24 \mathrm{~h}$ block-like boehmite crystals (ca. $4 \mu \mathrm{m}$ in size) filled and sealed the open cracks. This was reflected in an increase in pore resistance up to two orders of its magnitude compared with that of cleaved coatings without fillers [68].

\subsubsection{Viscoelastic Recovery and Healing}

Micro-damage self-healing also exists in asphalt mixtures. After the removal of external load, two processes occur: the first is viscoelastic recovery in the bulk of the material and the second is healing in a fracture process zone [69]. Viscoelastic recovery occurs in the bulk of the material only after a stress or strain is induced which is sufficiently large to generate damage. The phenomenological difference between viscoelastic recovery and healing is that the former is due to the rearrangement of molecules within the bulk of the material, whereas the latter is due to the wetting and inter-diffusion of material between the two faces of a micro-crack to achieve properties of the original material. The three primary steps in the healing process are:

1) Wetting of the two faces of a micro-crack

2) Diffusion of molecules from one face to the other

3) Randomization of the diffused molecules to reach the level of strength of the original material

Again, especially rest periods introduced at certain damage levels increase the fatigue life of asphalt binders [69].

A very recent example of self-healing via reformation of weak, fracture interrupted bonds has been demonstrated on a supramolecular rubber employing molecules that associate together to form both chains and crosslinks via hydrogen bonds [70] (see Figure 9).

By mixing ditopic and multitopic molecules, which are able to associate with more than two other molecules, a network could be formed. The system shows recoverable extensibility up to several hundred per cent and very little creep under load. In contrast to conventional cross-linked or thermo-reversible rubbers, these systems, when broken or cut, can be simply repaired by bringing together fractured surfaces in contact-an example of autonomous self-healing at room temperature. The process of breaking and healing can be repeated many

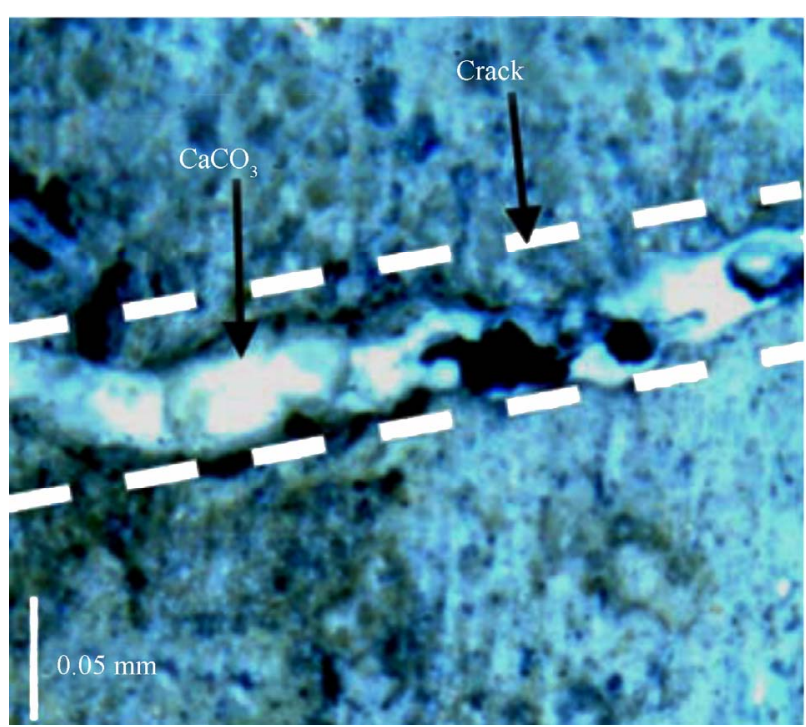

Figure 7. Crack blockage in mortar specimen by $\mathrm{CaCO}_{3}$ after $\mathrm{NaCl}$ solution exposure, crack width $=49$ microns. Reprinted from Cement and Concrete Research, Permeability and self-healing of cracked concrete as a function of temperature and crack width, 33, H.-W. Reinhardt, M. Jooss, 9131-9136, Copyright (2003), with permission from Elsevier. 

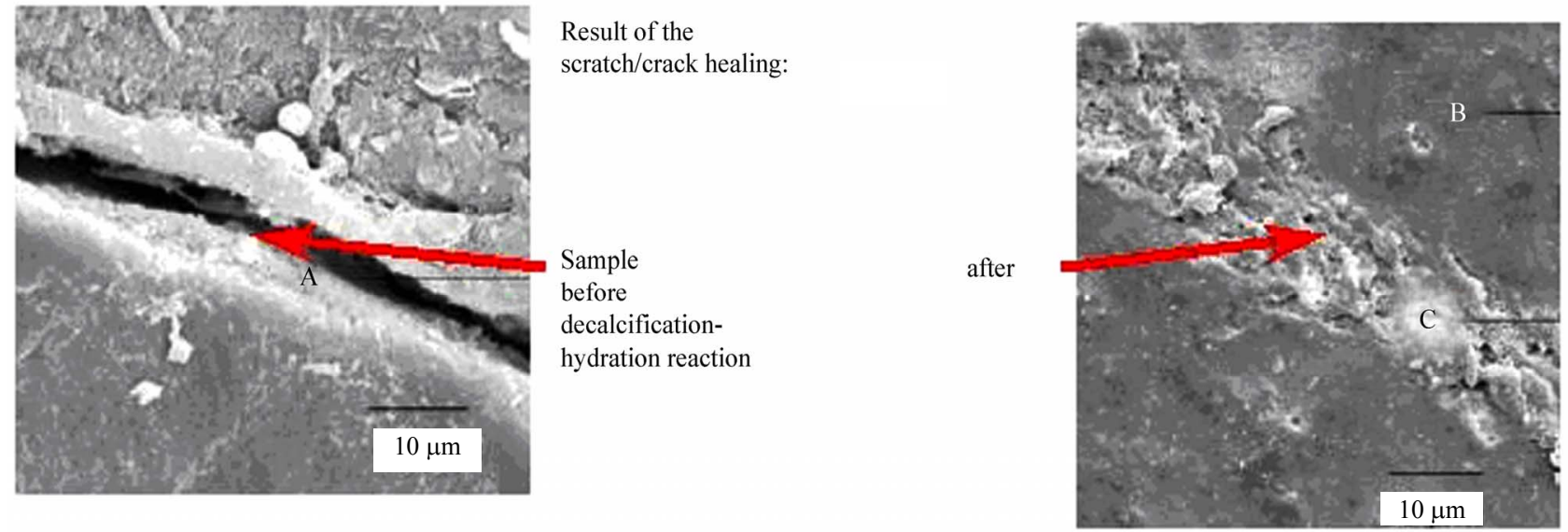

Figure 8. Self-repair action of a decalcification-hydration reaction on a polymer composite surface [68].

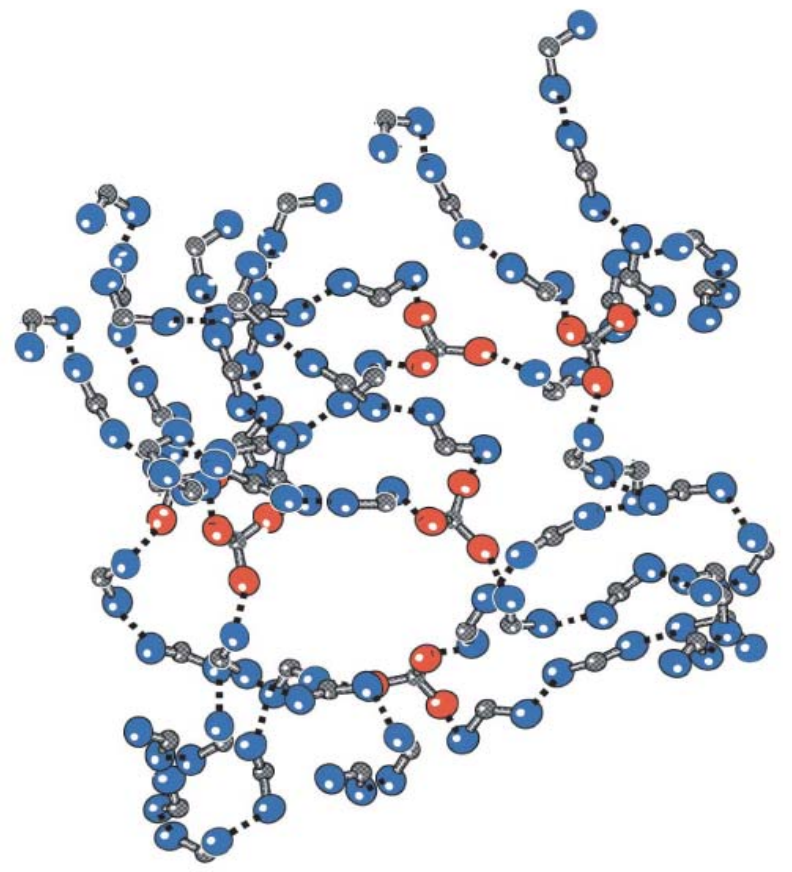

Figure 9. Schematic view of the Supramolecular reversible network formed by mixtures of ditopic (blue) and tritopic (red) molecules associated by directional H-bonds (represented by dotted lines) as employed for the self-healing rubber. Reprinted by permission from Macmillan Publishers Ltd: [Nature] (Self-healing and thermoreversible rubber from supramolecular assembly, P. Cordier, F. Tournilhac , C. Soulie-Ziakov, L. Leibler, 451, 977-980), copyright (2008).

times. However, the time needed to establish a contact of the fractured surfaces determines the healing efficiency. Longer healing times lead to better healing, but even when contact time is as short as fifteen minutes a repaired sample can be deformed up to about $200 \%$ without breaking.
In order to be self-mending, the supramolecular rubber has to be made from small molecules and the supramolecular associations have to be strong and longlived so that at equilibrium, the fraction of non-associated groups in the network is low. However, the strength of the associations has to be lower than that of covalent bonds so that, when broken, many non-associated groups are present near the fracture surface. Self-healing is efficient because a large number of groups 'eager' to link is available. At shorter healing times, fewer bridges across the interface are formed and the elongation at break is lower. When the sample is not mended immediately after being broken, the number of non-associated groups available for healing progressively decreases as they find other partners for $\mathrm{H}$-bonds and become unavailable for a re-formation of the entangled network.

\subsubsection{Self-Healing by Phase Changes and Volume Expansion}

The self-healing potential in metals and metal alloys is connected with a dynamic precipitation of atoms and the consequent potential to delay crack initiation and crack propagation as observed in $\mathrm{Al}$ alloys similar to a plasticity-induced crack closure. When a dislocation interacts with a precipitate, solute atoms will be transferred to the precipitate, causing Ostwald ripening, if their binding energy to the precipitate exceeds that to the dislocation. Conversely, solute atoms will be removed from the precipitate if their binding energy to the dislocation is greater. Dynamic precipitation tends to compensate for micro structural damage, with the result that a higher integrity structure is maintained for an extended lifetime [71]. Alternatively, the precipitation of dissolved atoms like $\mathrm{B}$ and $\mathrm{N}$ under formation of $\mathrm{BN}$ on creep cavity surfaces (in for example austenitic steel) leads to a self-healing of the creep cavitations together with an increase in creep rupture strength and ductility [72]. 
Oxidative self-recovery of surface damaged ceramics and ceramic composites may also be a useful self-repair mechanism (Figure 10). Oxidation of $\mathrm{NiAl} / \alpha-\mathrm{Al}_{2} \mathrm{O}_{3}$ composites provides the metamorphic surface layers consisting of $\mathrm{NiAl}_{2} \mathrm{O}_{4}$ and $\mathrm{Al}_{2} \mathrm{O}_{3}$ with compressive stress to improve the mechanical properties [73]. The peculiarity of this material is the dominant formation of $\mathrm{NiAl}_{2} \mathrm{O}_{4}$ along with the grain boundary of the matrix $\mathrm{Al}_{2} \mathrm{O}_{3}$ by the reaction,

$$
2 \mathrm{NiAl}+\mathrm{Al}_{2} \mathrm{O}_{3} \text { (boundary) }+5 / 2 \mathrm{O}_{2}=2 \mathrm{NiAl}_{2} \mathrm{O}_{4}
$$

The rapid diffusion of the reaction product through the grain boundary results in the repair of surface defects (see Figure 10).

This concept has been further developed for thermal barrier coatings with a composition of NiCrAlY, again with $\mathrm{Al}$ as reactive element. This enables a reaction with oxygen to form a protective barrier layer under volume expansion in response to damage due to thermal mismatch during thermal cycling. Here, an addition of 0.1 $0.2 \% \mathrm{Zr}$ leads to an optimisation of the self-repair process [74]. Similar concepts were reported for the systems $\mathrm{ZrB}_{2} / \mathrm{SiC}$ [75], $\mathrm{SiC} / \mathrm{Al}_{2} \mathrm{O}_{3}$ [76], $\mathrm{SiC} / \mathrm{Si}_{3} \mathrm{~N}_{4}$ [77], $\mathrm{BN} / \mathrm{SiC}$, $\mathrm{B}_{4} \mathrm{C}, \mathrm{SiC} / \mathrm{B}_{4} \mathrm{C}, \mathrm{MoSiC} \mathrm{C}_{2}$ containing ceramics and $\mathrm{Ti}_{3} \mathrm{AlC}_{2}$ [78], where the surface crack oxidation leads to an effective volume expansion and, thus, to a filling of the crack opening.

Volume-expanding phases are of course most suited to be applied in self-healing systems, since they offer the possibility to fill the empty crack volume completely and to re-generate the structural integrity of the system. This requires a material identically or similar in properties to the damaged matrix material. Examples include phase transition tetragonal-monoclinic (e.g. in $\mathrm{ZrO}_{2}$ ) phase transitions, $\alpha-\beta-\gamma$ phase transitions as seen in sulphur, TRIP transformation, piezo-effects, curie-transitions and others which induce a volume change of $1-4 \%$, sufficient to fill cracks and to perform a healing operation. Those changes are mostly triggered by a critical strain $\sigma$ or/and by temperature. More simply is the healing of zirconia and especially yttria-stabilized zirconia (YSZ) where an efficient damage recovery in large-scale molecular dynamics simulations has been observed [79]. Dynamic annealing is highly effective in zirconias during the first 5 ps of damage evolution, especially in and due to the presence of oxygen structural vacancies. The number of anion displacements in YSZ keeps increasing with time instead of reaching a steady value as in $\mathrm{ZrO}_{2}$. This demonstrates the role played by structural vacancies in the migration of $\mathrm{O}^{2-}$. Defect diffusion plays an importantrole in the dynamic recovery of radiation damage. Espe-

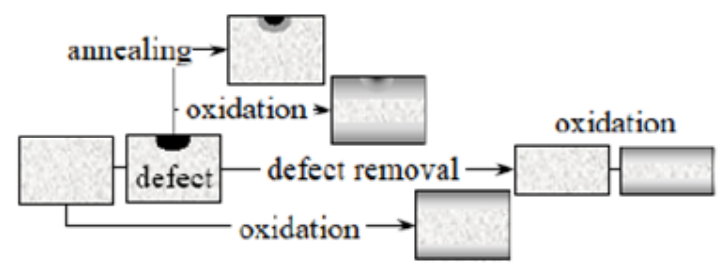

$$
2 \mathrm{NiAl}+5 / 2 \mathrm{O}_{2}=\mathrm{NiAl}_{2} \mathrm{O}_{4}+\mathrm{NiO}
$$

\section{Result of the crack healing:}
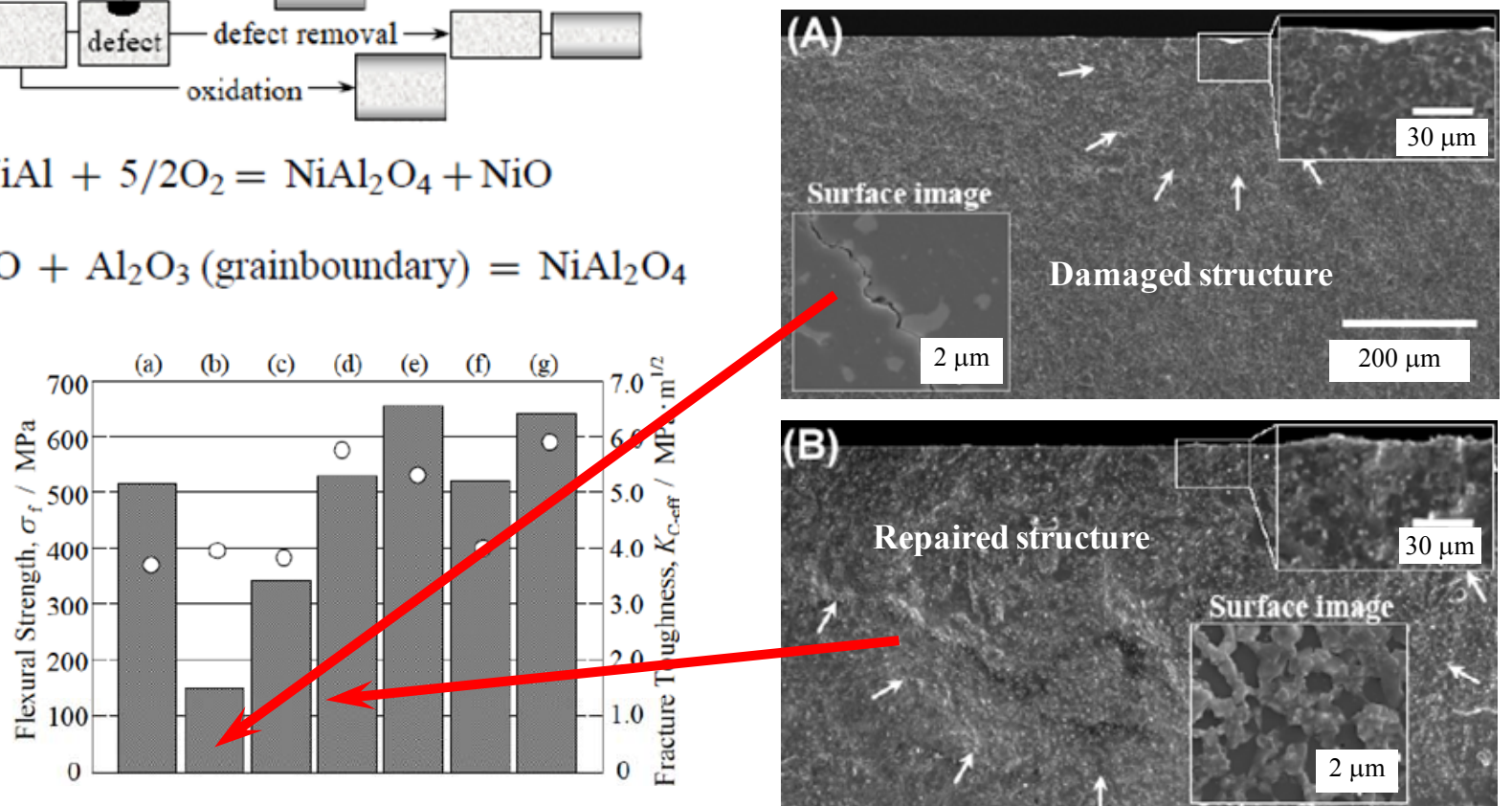

Figure 10. Crack healing of ceramics due to oxidative self-recovery of ceramics [73]. Flexural strength (a) without defect, (b) with defects, (c) annealed, (d) repaired, (e) removal of defect, (f) oxidized without defect, and (g) oxidized after removal and fracture surface of (A) with defects and (B) repaired materials, the symbol o corresponds to the right axis. 
cially in YSZ, the anion interstitials almost completely annihilate with structural vacancies, resulting in no residual radiation-induced defects on the anion sublattice (see Figure 11).

This results in near-complete recovery of damage. Damage recovery on the cation sublattice is assisted by the anion sublattice recovery, which explains the remarkable radiation tolerance of stabilized zirconia.

If those concepts are not applicable, a possible reaction with components provided by the environment using transport via the gas phase might be an option for a self-repair action. This principle is especially useful in coatings where dimension constraints prevent the use of other options for self-repairing design (see below). Coatings should not only protect a surface from a possible damage and/or corrosion but should also provide additional properties like anti-reflection, gas barrier etc. However, if coatings are damaged and/or pinholes formed, serious damage could occur due to corrosion on and in the substrate. To fill such damage areas and/or pinholes and to restore the passive corrosion protection or other functionalities, the coating can be loaded with nanoparticles, which react upon volume expansion with moisture or oxygen. In a practical example, raw smectic clay has been used as the phase being able to absorb moisture under volume expansion [80]. In a later study, this concept has been developed further leading to a true recovery of damaged surfaces [81]. There, the option of healing surface scratches in multi layer coatings consisting of a polysiloxane film on top of a thin montmorillonite layer as expandable phase was studied (see Figure 12). Filling of cracks in the polymeric topcoat occurred primarily within the first two hours of exposure to moisture-saturated air and resulted in a good restoration of the surface flatness [81].

\subsubsection{Mimicking Nature}

Mimicking nature has resulted in a variety of self-healing systems. One of the first synthetically design systems developed, tested and used in ceramics (concrete) and subsequently in polymeric matrices compromising all three functionalities (damage sensor, healing mobile materials, structurally integer functioning system) are resin filled glass capillaries incorporated in the matrix material [82-90]. Upon damage or fracture, the glass capillaries act as a strain sensor, releasing the encapsulated adhesive components (epoxy and hardener, cyanoacrylate etc.) into the composite structure to perform the repair action while (partially) filling the crack. The damage can be sensed/visualized by adding a fluorescent dye to the components (see Figure 13); the capsules (glass capillaries) also serve as part of the (reinforcing) composite structure.

This 'bleeding composites' approach thus combines damage detection with a high sensitivity due to the continuity of the hollow fibres and self-repair, however, also with two draw-backs: 1) they will release an excess of healing liquid since the volume of the compartments is often much larger than the damage volume and 2) they will display a tendency to clotting since the propagation of the repairing reaction of the stored agent within the hollow fibre can still occur thus preventing the chance of multiple repair.

Systems following a similar idea were developed for an application in thermosets (epoxy); the approach has been applied to other matrix materials as well [91-94].

Here, the resin containing containers are hollow polymeric capsules (see Figures 14,15).

The fragile capsules act as sensor and as reservoir of the resin. Upon fracture the released monomer reacts with the catalyst distributed within the matrix forming a cross-linked polymer network and repairing the damage.

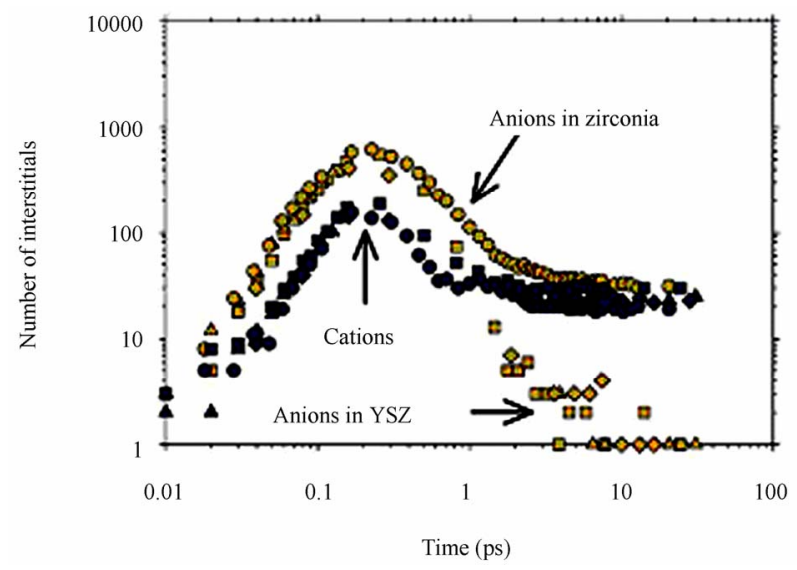

Figure 11. Evolution of the number of interstitials in YSZ and pure $\mathrm{ZrO}_{2}$ following $30-\mathrm{keV} \mathrm{Zr}$ recoils. The numbers of cation and anion interstitials in $\mathrm{ZrO}_{2}$ and YSZ peak at about 0.2 ps and then decline sharply. Subsequently, in YSZ, the anion interstitials almost completely annihilate with structural vacancies, resulting in no residual radiation-induced defects on the anion sublattice. The difference in anion sublattice damage between $\mathrm{ZrO}_{2}$ and YSZ is due to the presence of structural vacancies in YSZ. (squares, triangles, and diamonds represent [001], [110], and [111] recoils, respectively) and pure $\mathrm{ZrO}_{2}$ (circles) [79].

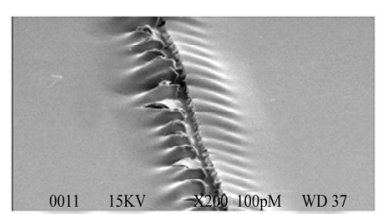

(a)

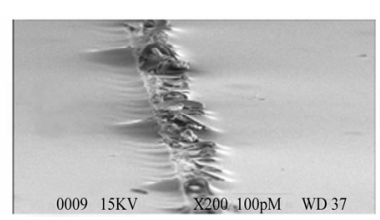

(b)
Figure 12. Crack-healing of multilayer coatings due to phase expansion upon moisture absorption before (a) and after $21 \mathrm{~h}$ of healing (b) in wet atmospheric conditions [81]. Scale bar $=$ 10 microns. 
The healing efficiency of this concept is high (see Figure 16) [95-97].

However, since a significant load of microcapsules is required to get efficient self-healing due to the small

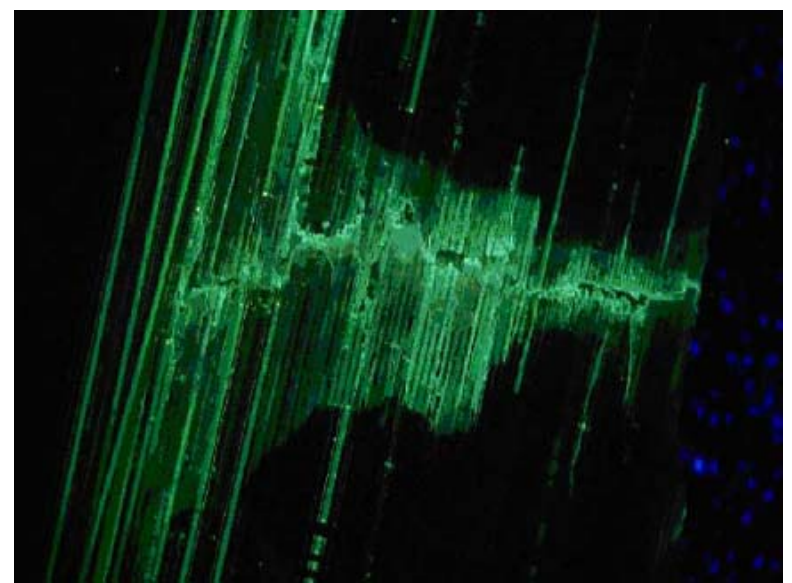

Figure 13. Fracture of a hybrid solid/hollow fibre reinforced plastic showing bleeding of UV fluorescent dye along crack paths(x 45 magnification) [88].

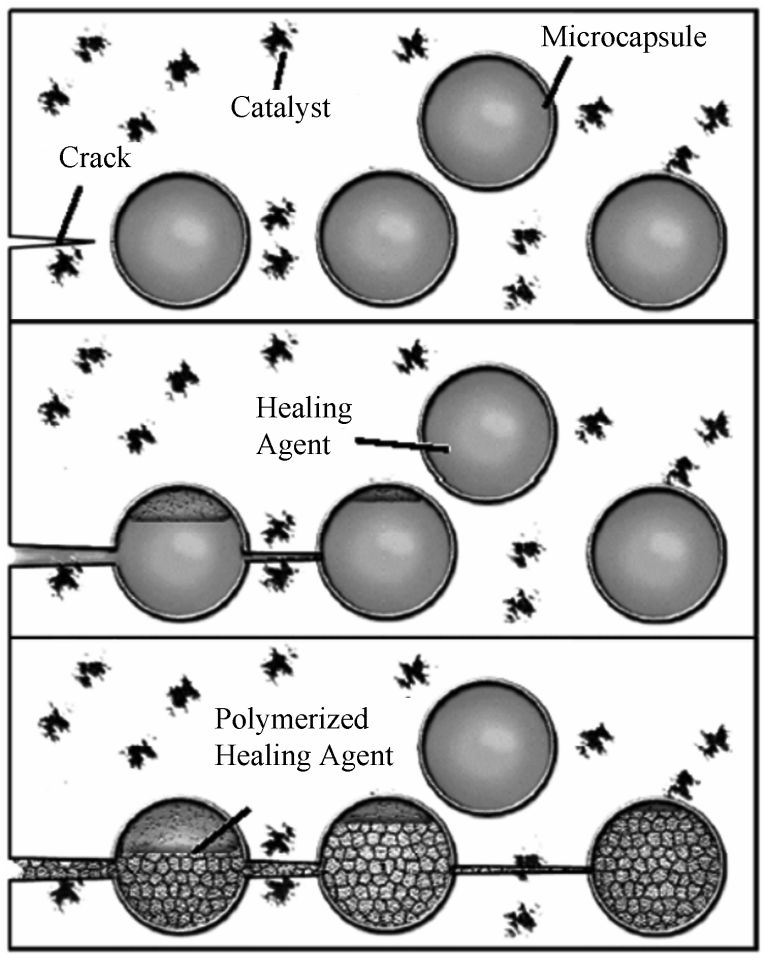

Figure 14. Schematic picture of the self-healing action of a in spherical capsules embedded resin upon damage/fracture of the matrix material. Reprinted by permission from Macmillan Publishers Ltd: [Nature] (Autonomic healing of polymer composites, S. R. White, N. R. Sottos, P. H. Geubelle, J. S. Moore, M. R. Kessler, 409, 794-797), copyright (2001)

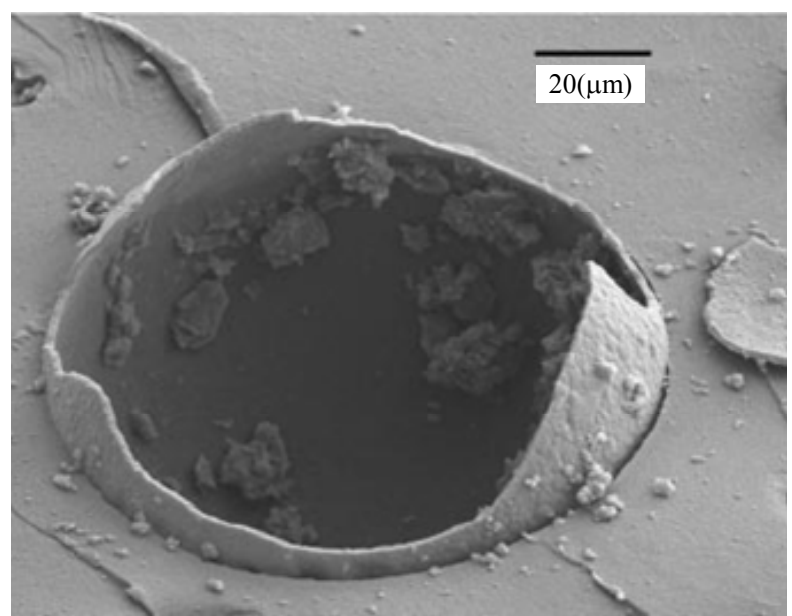

Figure 15. SEM picture of a broken capsule. Reprinted by permission from Macmillan Publishers Ltd: [Nature] (Autonomic healing of polymer composites, S. R. White, N. R. Sottos, P. H. Geubelle, J. S. Moore, M. R. Kessler, 409, 794 797), copyright (2001).

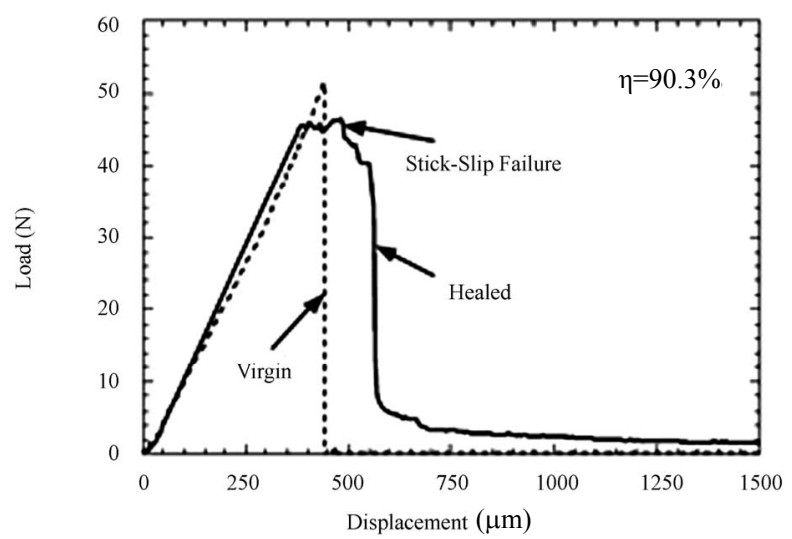

(a)

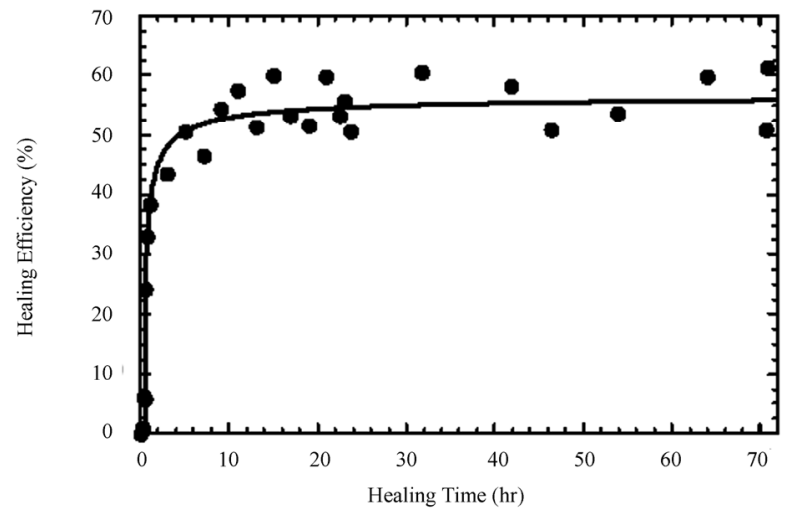

(b)

Figure 16. a) Healing efficiency of the capsule strategy in a single experiment and b) in time [95].

amount of liquid depleted upon fracture and the limited probability of fracture during damage, the mechanical 
properties of the matrix material are substantially weakened and limit the application of this concept. Furthermore several material processing aspects are also not favourable. Anyway, these systems have been further developed and optimized for coating applications [98-101] since they offer an autonomic self-repair for anti-corrosion protective coatings.

The latest developments of autonomous systems combine the concept of the incorporation of a self-healing liquid with the increase in mobility of macromolecules via solvent stimulation [102-104]. Here, solvent-containing micro-capsules were either embedded in an epoxy matrix or in a matrix of thermoplastic polymers. In the case of the thermoset, the presence of the solvent increases the mobility in the system and healing efficiencies of up to $82 \%$ was observed due to molecular diffusion and reaction of residual functionality [102, 103]. The healing performance appears to be inversely related to cross-link density. Healing with epoxy-solvent microcapsules is superior to capsules that contain solvent alone (up to $120 \%$ healing efficiency), and data showing multiple healing events are reported for this system. In the case of the thermoplastic matrix, the mobility of the macromolecules increases due to the lowering of the glass transition temperature via solvent chain interactions leading to the possibility of re-entanglement formation and complete crack closure (see Figure 17) [104].

In order to combine the advantages of the two existing concepts for the storage of a self-healing liquid (the hollow fibre container and the spherical capsule concept), experiments have been carried out to develop anisotropic capsules [105]. Such particles offer a better probability of being fractured upon damage than spheres. Thus a substantial lower load of capsules (up to five times less) is needed for a realisation of self-healing properties and the initial mechanical properties of the system will be preserved [105]. On the other hand, they offer the same advantages with respect to processing and preparation as the spherical capsules compared to the hollow fibres.

Ultimately, a mimic of the blood vessel system capable of a repeated, autonomic repair of damaging events seems a perfect self-healing material system. Healing in such systems is accomplished by a vascular network system supplying the necessary components at the damage site. A vascular system permits a continuously and repeatable repair of all types of failure modes at any point in the structure since it renews the supply of the healing part of the system during the lifetime of the structure at any location and any time.

A skin structure exhibiting flexibility, self-healing and damage sensing is the simplest system realizing a network-like supply of an active self-healing liquid [106].
The skin is fabricated on a substrate of copper-clad polyimide sheets in a layer-by-layer technique using polyimide sheets and an ultraviolet (UV)-curable epoxy. The UV-curable epoxy is used as both a structural adhesive and as the self-healing fill material (see Figure 18). The skin structure is integrated with an array of inductor-capacitor (LC) circuits, where each circuit is characterized by a unique resonant frequency. If the skin is damaged, the UV-curable epoxy is released and is cured by ambient sunlight. Further, damage affects one or more of the LC circuits, altering its resonant frequency. An integrated antenna coil is used to detect and locate the damaged portion of the skin; so far tests indicated a good performance with respect to self-healing of the skin and fault isolation.

Other examples of such a system have been described by Toohey et al. [107,108] and by Williams et al. [109, 110]. Here, a 3-dimensional micro-vascular network system, either manufactured by a direct write assembly procedure using a fugitive ink or by connection of glass capillaries via risers has been embedded in an epoxy

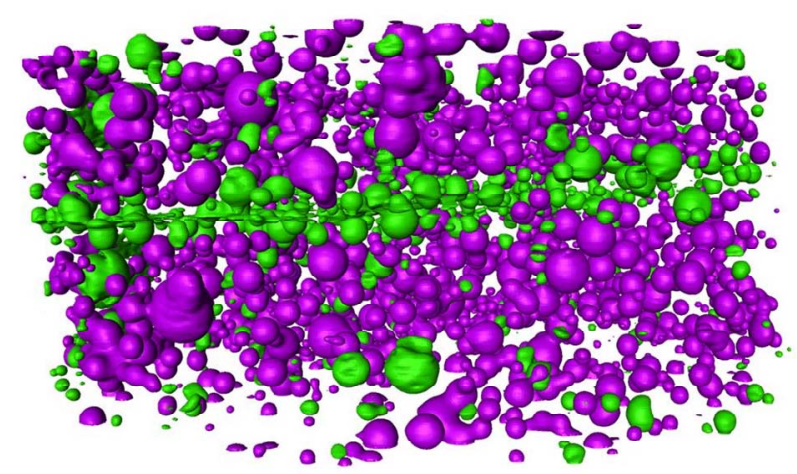

Figure 17. MRI tomogram of a healed thermoplastic containing solvent filled capsules. The green capsules are empty. The majority of the empty capsules are located at the fracture plane as intended, healing is obtained, and no empty space of the original fracture plain is observable [104].
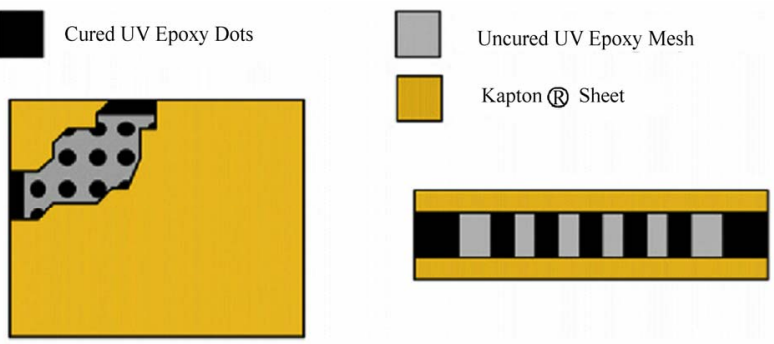

Figure 18. Basic diagram of self-healing skin, top view with cutaway and cross-section. Reprinted by permission from IOP Publishing Ltd: [Smart Mater. Struct.] (A flexible, self-healing sensor skin, J. A. Carlson et al, 15, N129), copyright (2006). 
matrix system and tested upon bending. These systems show repeatedly crack healing upon damage.

Alternatively, 3D microvasular networks can be realized simply by loading the hollow micro-fibres of a free-standing paper manufactured by electro-spinning with a healing agent displaying a rapid reactivity, volatility and ability to propagate repair triggered e.g. by the influx of moisture [111]. The self-healing agent used is $\mathrm{TiCl}_{4}$ blocking the pores of the hollow micro-fibres as well as sealing possible cracks by the formation of ceramic particles of $\mathrm{TiO}_{2}$ after hydrolytic decomposition in contact with water vapour.

The natural blood vessel system works, however, in a slightly different way [112]. In this case, a temporary repair as response of a physical damage is achieved in the form of a clot that plugs the defect. During subsequent days steps to regenerate the missing parts are initiated. The healing of a skin wound is a complex process requiring the collaborative efforts of many different tissues and cell lineages. Inflammatory cells and then fibroblasts and capillaries invade the clot to form a contractile granulation tissue that draws the wound margins together; meanwhile, the cut epidermal edges migrate forward to cover the denuded wound surface. The formation of a clot then serves as a temporary shield protecting the denuded wound tissues and provides a provisional matrix over and through which cells can migrate during the repair process. The clot consists of platelets embedded in a mesh of crosslinked fibrin fibers derived by thrombin cleavage of fibrinogen, together with smaller amounts of plasma fibronectin, vitronectin, and thrombospondin.

An interesting analogy to the process uses nanoparticles dispersed in polymer films and shows in simulations and experiments a preferred coagulation of nanoparticles at areas of stress concentration similar to the clotting of blood platelets at zones of damage [113-116]. This is because the mobility of the filler particles at the length scales present in nanocomposites is controlled by the conformational entropy of polymer chains. Polymer chains close to nanoparticles are stretched and extended, which results in an entropy penalty. This decrease in polymer conformational entropy is greater than the decrease in nanoparticle translational entropy; thus, nanoparticle-polymer interactions are minimized by segregation of the nanoparticles in areas of stress concentration such as a crack tip. For particles comparable to the radius of gyration of the polymeric chain, the decrease in conformational entropy of the respective polymer sub-chains upon particle sequestration is dominant; the chains gain conformational entropy while repelling the particles because they do not have to stretch around particles [113-115]. The system can relieve the entropic cost of chain stretching by allowing the particles to self-assemble to the solid walls. In the presence of a notch, the nanoparticles are driven to localize in the notch. The driving force for this localization is a polymer-induced depletion attraction; the confined polymers in the melt gain conformational entropy by "pushing" the fillers to the surfaces and into the notch (Figure 19).

The time required for the particles to migrate to the notch is comparable to the time needed for the chains to move by approximately four radii of gyrations. The morphology obtained from the simulation allowed a determination of the mechanical properties of the nanocomposite-coated surface. The calculations show that the nanoparticle fillers significantly reduce the stress concentration at the notch tip relative to the case where the notched surface is just coated with a pure polymer layer (see Figure 20).

As a consequence, load transfer from the matrix to the agglomerated nanoparticles is predicted and the mechanical properties can recover to $75 \%$ of their original value [114]. The calculations on the crack tip opening displacement indicate that the presence of the nanocomposite in the notch would inhibit the system from undergoing further damage (crack propagation from this notch) when an external load is applied to the system. It is important to realize that some fractions of the polymer are also localized in this damaged region. If the macromolecules and the particles are chemically compatible, the chains provide cohesion between the fillers and the polymer coating.

The application of such nanocomposite coatings could thus constitute an important step in the production of components with defect-free surfaces. Upon appearance of a defect, the coating effectively senses its presence and then causes the repair of the damaged area.

In a practical example, nanoparticles are added to a high viscous liquid polymer that is sandwiched between two brittle, glassy layers (see schematically in Figure 21).

This architecture is common in multi-layer composites that are used in optical and anticorrosion coatings, mi-

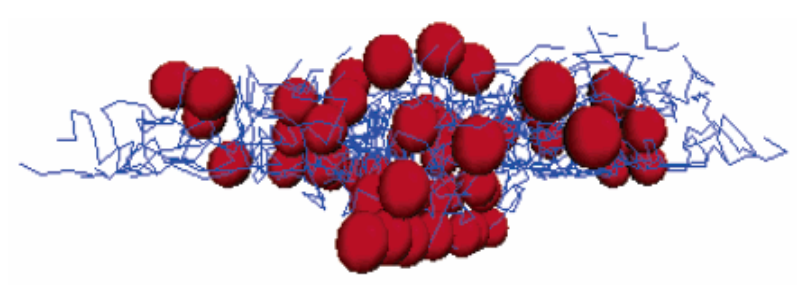

Figure 19. An instantaneous molecular configuration showing a surface notch filled with nanoparticles due to a depletion attraction between the particles and the surface. Particle beads are shown as spheres and polymer chains as lines [115]. 


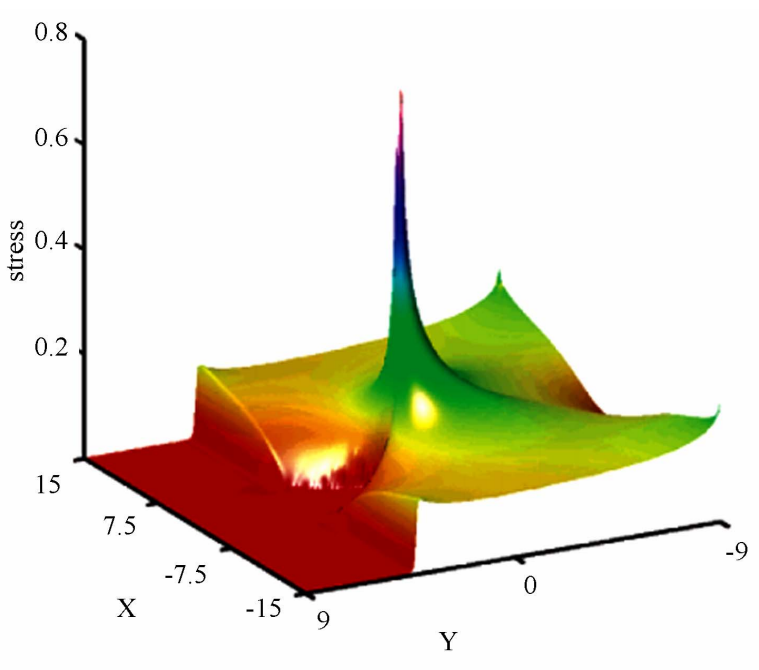

(a)

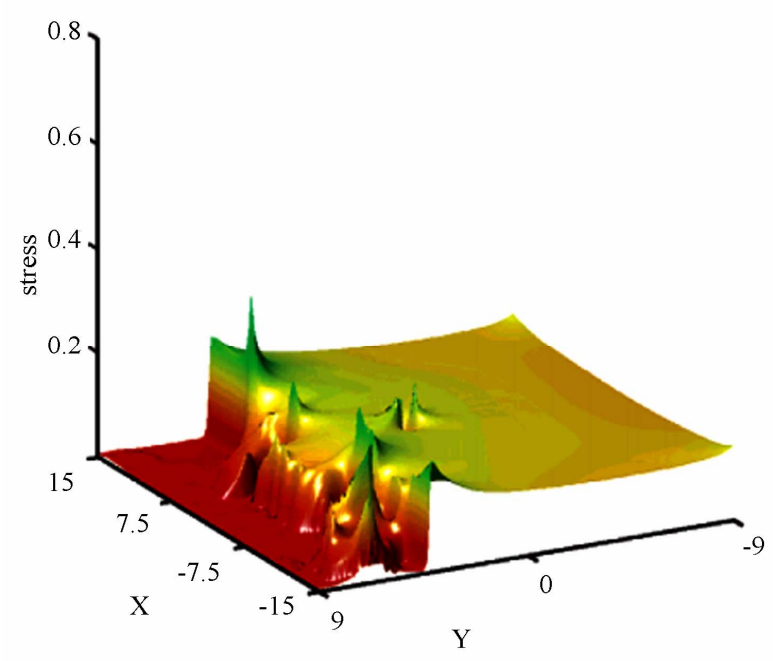

(b)

Figure 20. Results of the calculation of the stress distribution using a lattice-spring model for (a) a polymer coating not containing nanoparticles. The stress reaches a maximum at the notch tip. (b) The polymer coating contains nanoparticles and the stress at the notch tip is significantly reduced. The colour bar indicates the magnitude of the stress [114].

croelectronics packaging, and solid-state devices. Such films are susceptible to failure through the formation of cracks, which propagate vertically to the polymer layer. Since the polymer is fluid-like it expels the nanoparticles to the brittle surfaces, where some of the particles pack into the cracks, effectively mending the brittle surface. In the experimental study [117], the particles were fluorescent and could readily be visualized in the cracks (see Figure 22).

In this example, the polymers and particles provide the healing mechanism without any external intervention.
Brittle film

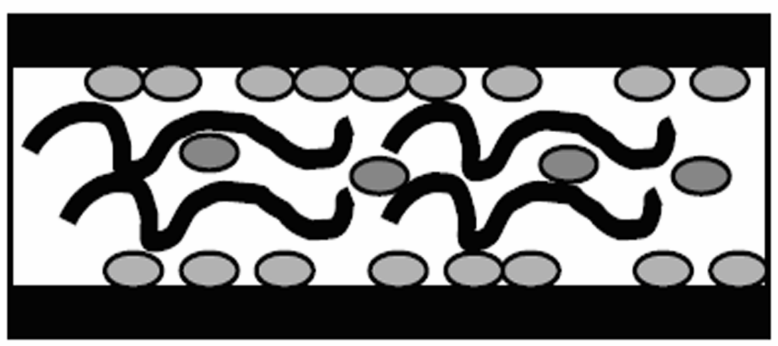

Crack

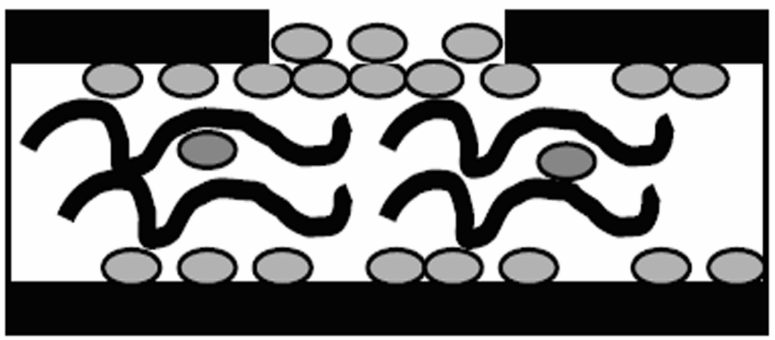

Figure 21. Scheme of a multilayer composite (coating). A polymer layer (represented by the black chains) containing nanoparticles is sandwiched between two intact, brittle layers. Upon appearance of, a crack in the top surface and due to the particle-polymer interactions, the particles become localized in the crack and effectively mend the damage.

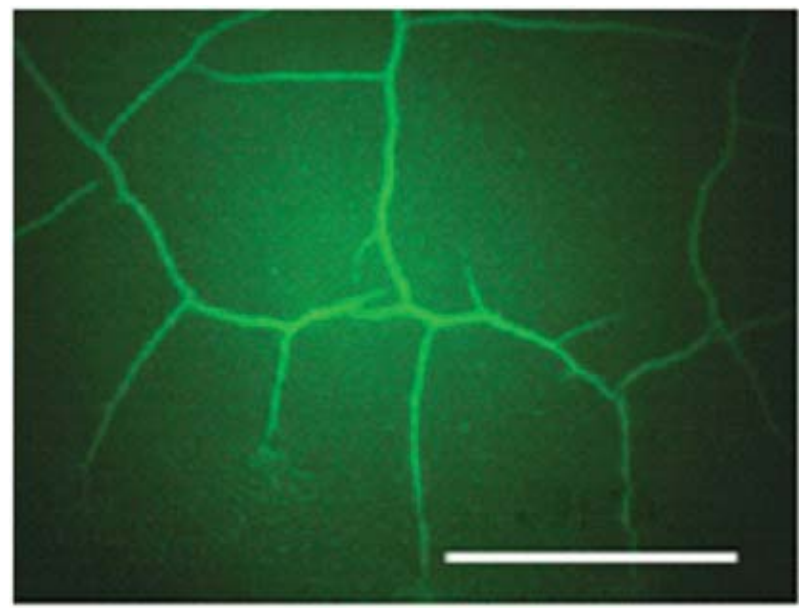

Figure 22. Fluorescence microscope image of a crack in a 60 nm SiOx layer on a mixture of PMMA with PEO-covered 3.8-nm-diameter $\mathrm{CdSe} / \mathrm{ZnS}$ nanoparticles. The cracked film is viewed with a fluorescence microscope, in which the segregation of the CdSe nanoparticles to the cracks is highlighted by the fluorescence of the nanoparticle. [117], scale bar $=50 \mathrm{mi}$ crons. Reprinted by permission from Macmillan Publishers Ltd: [Nature Materials] (Entropy-driven segregation of nanoparticles to cracks in multilayered composite polymer structures, $\mathrm{S}$. Gupta , Q. Zhang, T. Emrick, A. C. Balazs, T. P. Russell, 5, 229-233), copyright (2006). 
The approach has the advantage of being "re-usable"; when new cracks appear, the polymers again drive the particles to the damaged site and thereby continue the repair. In principle, this process can continue until essentially all the particles in the polymer layer are expelled and localized in the cracks.

Finally, since the particles can potentially be made out of the same materials as the brittle walls, the system will resemble the initial, undamaged materials. Additionally, the fluorescing particles provide a useful diagnostic tool, pinpointing the location of the cracks and revealing the mechanical state of the material.

A model has been published describing the rolling motion of a fluid-driven, particle-filled microcapsule along a heterogeneous, adhesive substrate to show how the release of the encapsulated nanoparticles can be harnessed to repair damage on the underlying surface [118]. The microcapsules that could act as 'artificial leukocytes' are driven by an imposed flow to move along an adhesive surface, which represents the wall of a microchannel (either in the synthetic microvasculature, or more generally, in a microfluidic device). The microcapsules enclose a solution of nanoparticles and these nanoparticles can diffuse from the interior of the capsule into the host fluid. When the capsule is trapped at the leading edge of the damaged region, a relatively high fraction of the released particles are now localized near this region and can more effectively cover this damaged site. Once the damage is repaired, the capsule can again be driven by the imposed flow to move along the surface where they could potentially sense and perform the repair action at another damage site.

So far, all self healing mechanisms and discussed systems tried to mimic nature by filling or re-filling cavities of damaged materials just like it is the case in nature. However, nature has more than one strategy to deal with threads. Continuously re-shaping, metabolic cycles as well as isolation of sub-critical thread concentrations (bacteria) are also healing strategies in nature.

Such metabolic reactions can also be used in polymeric systems to accompany a self-healing process as described for polycarbonate (PC, polyetherketone (PEK) and poly-p 2.6 dimehylphenylenether (PPE) [119]. In the last case e.g., the polymeric chains cut by heat, light, oxygen and external mechanical force will produce a radical on the end of the scission chain in the first step. Subsequently, a hydrogen donor stabilizes the radical. The Cu (II) catalyst added beforehand forms a complex with each end of the two different chains and withdraws two electrons from them. The chains combine, eliminating two protons from the ends and reducing the copper $\mathrm{Cu}$ (II) to $\mathrm{Cu}$ (I). The $\mathrm{Cu}$ (I) migrates in the polymer and reacts with an oxygen molecule in an oxidation step to $\mathrm{Cu}$ (II). The oxygen ion then reacts with two protons to form a water molecule which leaves the system. Key factors for the speed of the self-repair action are the concentration of the chain ends and the mobility of the chains (the recovery rate increases with decreasing initial molecular weight and increasing amount of dimethylphthalate as plasticizer), the oxygen partial pressure in the surroundings and the speed of emission of water (see Figure 23) [119].

The isolation of subcritical damage and the stop of further growth is the strategy applied while introducing

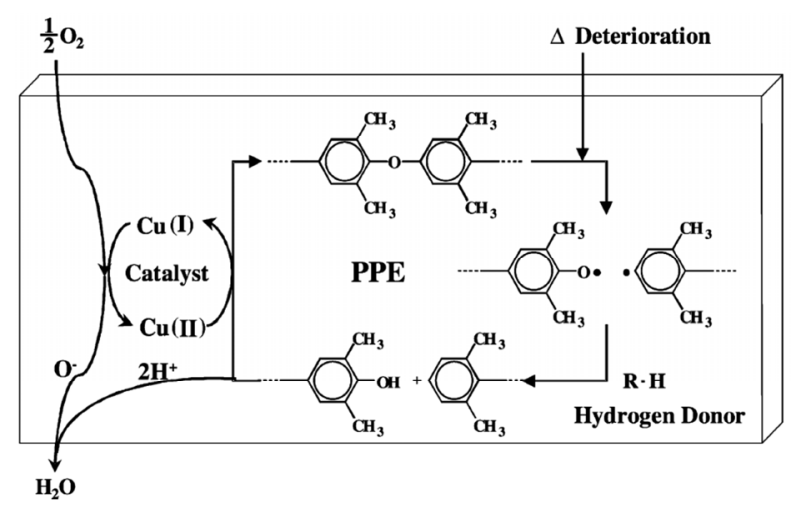

Figure 23. Scheme of the self-repairing metabolism in PPE. Reprinted by permission from IOP Publishing Ltd: [Sci. Technol. Adv. Mater.] (Self-repairing mechanism of plastics, K. Takeda et al, 4, 435), copyright (2003).
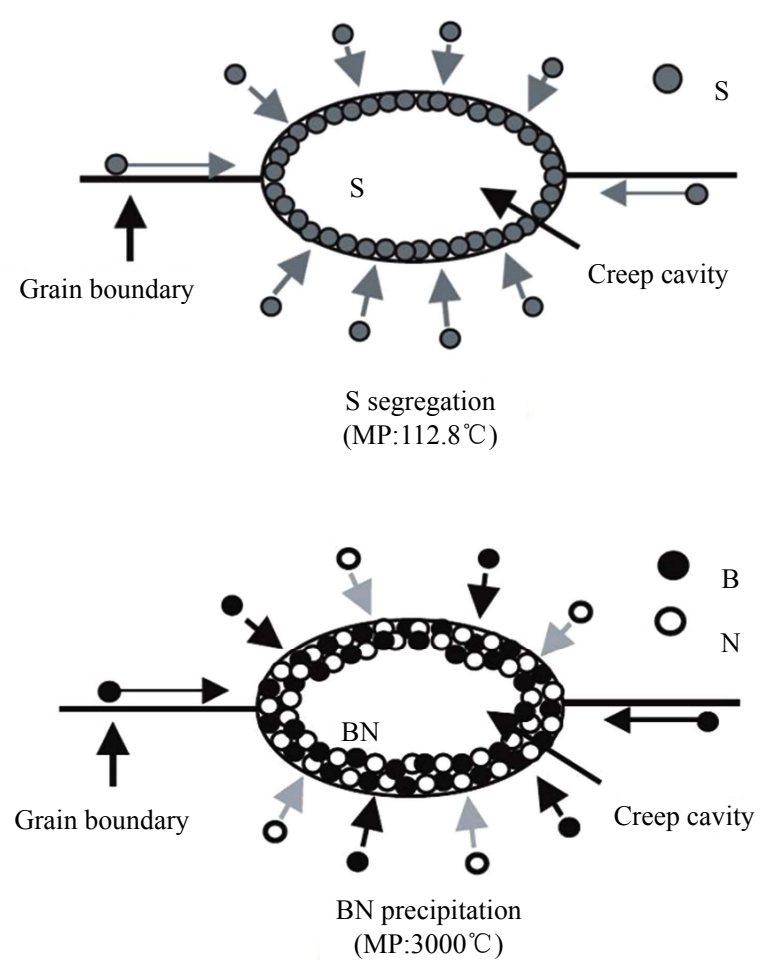

Figure 24. An illustration of $\mathrm{S}$ segregation and $\mathrm{BN}$ precipitation on creep cavity surface [120]. 
self-repair to austenic stainless steel [120] (see Figure 24). High temperatures during use of austenic steels leads to low ductility creep fracture in long timescales caused by the nucleation, growth and coalescence of grain boundary cavities. Dissolved sulphur segregates on cavity surfaces easily, and enhances the creep cavitation remarkably most likely due to a lowering the creep cavity surface energy and increasing the surface diffusion rate. The additions of $\mathrm{Ce}(0.016 \mathrm{wt} \%)$ is highly effective in removing the traces of soluble $\mathrm{S}$ in the steel through the formation of $\mathrm{Ce}_{2} \mathrm{O}_{2} \mathrm{~S}$. In the absence of $\mathrm{S}$ segregation, $\mathrm{B}(0.07 \mathrm{wt} \%)$ and $\mathrm{N}(0.007 \mathrm{wt} \%)$ can segregate due to their small atomic diameter efficiently to the creep cavity surface and form boron nitride $(\mathrm{BN})$ compound on the surface during creep. As BN is very stable at high temperatures, the precipitation of $\mathrm{BN}$ film on the creep cavity surface suppresses the creep cavitation in steel by reducing the creep cavity growth rate. The $\mathrm{BN}$ precipitation continues during creep exposure and heals the creep damage. The creep cavities are self-healed by a continuous precipitation of BN on creep cavity surface during creep exposure, and the growth of the self healed creep cavities is suppressed almost completely. The function of self-healing for creep cavitation provides the steel with a longer rupture life and higher rupture ductility.

\section{SENSORS AND TRIGGERS FOR SELF-HEALING SYSTEMS}

Fundamental to the understanding and implementation of self-healing systems is the detection of damage and a trigger mechanism which initiates the repair action. This is done in nature efficiently e.g. in the case of woundhealing by growth factors and matrix components that are available to provide these "start" signals, triggering relatively sedentary cell lineages at the wound margin to proliferate, to become invasive, and then to lay down a new matrix in the wound gap [112].

However, the tricky part is really the distinction between the healthy and the damage status of a given system. Here, a decision between acceptable damage and unacceptably changed behaviour of the system has to be made, or in other words an indication of the time of the usual transition from a normal state to a degraded state when attacks become successful and/or faults begin to take effect. In the end, any system should detect failure in a timely manner and must be smart enough to compute the degree of malfunction in the system and to asses finally whether the system actually needs the intervention by a "recovery program".

As stated above, sensor elements are essential for the design and functionality of self-healing systems. In the case of the hollow glass capillaries or the urea-formal- dehyde capsules used for the storage and delivery of a liquid performing the self-healing action, the shell of the capsule or the glass capillary itself act as strain sensor. They are tuned by their mechanical properties to break and to release the fluid in the event of damage.

The self-healing system based on re-formation of covalent bonds during a heating-cooling cycle fulfils in itself the sensor function. The low viscous system bridges the gaps of crack or damage due to the surface tension properties of the liquid.

However, it is certainly preferable to get access to autonomic self-healing systems with a more sophisticated embedded structural health monitoring sensor unit enabling decision making about the place and time of the required repair action to be taken. There are a number of possible damage sensors available, which are discussed with respect to their advantages and performance in [121]. Most important is the size and/or the location of the damage that can be resolved by a certain sensor system (see Figure 25), the size of the sensor itself and the requirements to get a certain sensor functioning (power consumption).

A combination of vibration and wave propagation data has been used to determine the location and degree of damage in structural components in an automated damage identification technique requiring minimal operator intervention [122]. To build such a detection system, a structure had to be instrumented with an array of actuators and sensors to excite and record its dynamic response. In order to determine structural damage, a damage index, calculated from the measured dynamic response of the structure in a reference state (baseline) and the current state, was introduced.

While the vibration-based analysis was used to identify widespread damage within the structure, the analysis of the waveform signals provides detailed information on the location and nature of smaller defects. The unified computer-assisted automatic data analysis procedure can improve the reliability of the defects detection capability and aid in the development of in-situ health monitoring systems for defects-critical structures. In a similar way, electrical resistance measurements are applied to monitor the health situation of a certain system using a (percolated) conductive network or carbon fibres incorporated in a structure. The resistance changes irreversibly upon damage, as shown for damage inflicted by flexure, tension, fatigue, and impact. The oblique resistance, as measured at an angle between the longitudinal and through-thickness directions, is particularly sensitive. This enables the real-time monitoring of damage in form of fire breakage and delamination in the case of composites tested in tension fatigue, compression and impact and provides an estimate of the remaining fatigue 


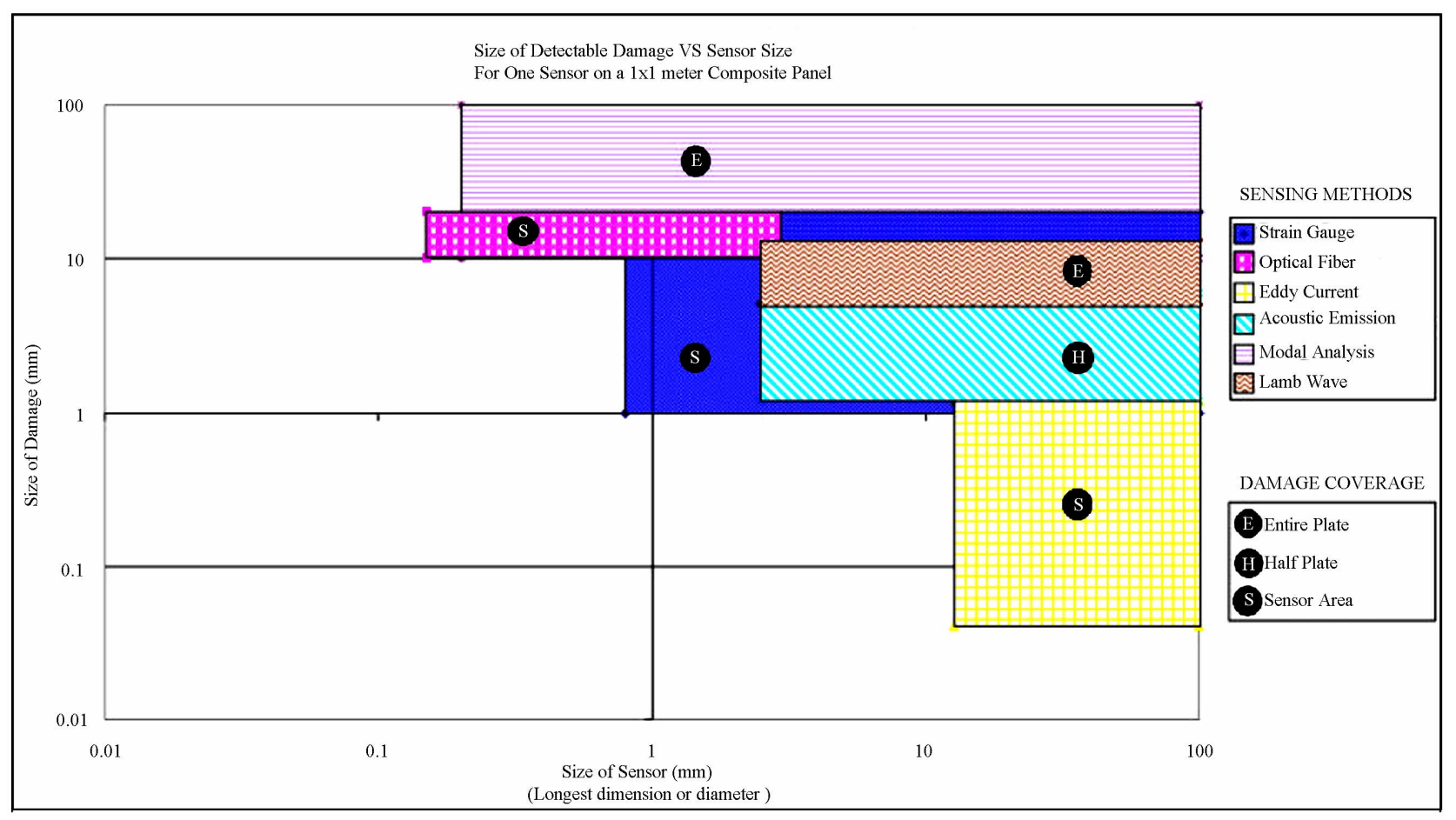

Figure 25. Sensor selection space comparing size of detectable damage with sensor size for various sensing methods [121].

life-time [123].

Also, carbon nanotube networks have been employed for sensing of distributed strain and damage and consequently for lifetime prediction and initiation of healing $[124,125]$. Under static load, changes in resistance with deformation and the initiation of microcracking during loading of the composite laminate can be monitored (see Figure 26).

On unloading, the resistance decreases to nearly the original value as the transverse cracks were closed by the stiff outer plies of the composite system. Upon reloading, the specimen show a sharp increase in resistance at much lower levels of deformation corresponding to reopening of the microcracks, indicative of permanent damage to the composite. This approach may be useful in self-healing systems.

In a recent study, the sensor possibilities of carbon nanotube networks incorporated in composite systems with respect to fatigue-induced damage was investigated with regard to impact on in-situ health monitoring, damage prognosis and the success of self-healing [126]. Monitoring of the volume and through-thickness resistance enabled a determination of the extent and propagation of fatigue-induced damage such as crack and delamination growth in the vicinity of stress concentrations. The conductive nanotube network also provides opportunities to repair damage by enabling fast heating of the crack interfaces; up to $70 \%$ recovery of the strength of the undamaged composite has been achieved. Again, the repair action has been taken by an enhanced mobility of a resin at temperatures above $T_{\mathrm{g}}$.

Anyway, the first step to autonomic self-repair is certainly structural health monitoring. Since all materials contain inherent defects, the detection of existence and location of damage requires a comparison between two system states. Sensors cannot measure damage directly, features need to be extracted through data processing and in a learning process type of damage and severity of damage can be identified.

Therefore, e.g. machine learning for structural health monitoring has to be applied. In general, there are two approaches to damage identification [127]. Model-driven methods establish a high-fidelity physical model of the structure, usually by finite element analysis, and then establish a comparison metric between the model and the measured data from the real structure. If the model is for a system or structure in normal (i.e. undamaged) condition, any departures indicate that the structure has deviated from normal condition and damage is inferred. Data-driven approaches also establish a model, but this is usually a statistical representation of the system, e.g. a probability density function of the normal condition. Any departures from normality are then signalled by measured data appearing in regions of very low density. The algorithms that have been developed over time for data-driven approaches are mainly drawn from the disci- 


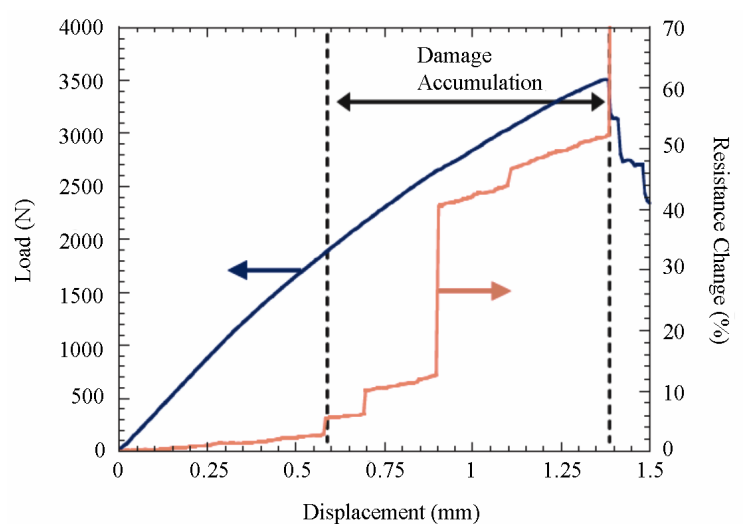

Figure 26. Load-displacement and resistance curves for a composite system with incorporated carbon nanotubes acting as strain sensors [124]. A linear increase in resistance with load is clearly seen. Upon the initiation of microcracking there is a sharp change in the resistance. In the progression from the first initiation of cracking to ultimate failure of the composite laminate the resistance changes drastically. Thostenson, E.T., Chou, T.-W., "Carbon nanotube networks: Sensing of distributed strain and damage for life prediction and self healing", Advanced Materials, (2006) 18 (21), pp. 2837-2841 Copyright Wiley-VCH Verlag GmbH \& Co. KGaA. Reproduced with permission.

pline of pattern recognition, or more broadly, machine learning. Apart from the first step to structural health monitoring, the detection of damage including data capture, feature selection as a process of amplification and discarding of redundant information and novelty selection, the damage location and the damage assessment which need as additional part a network of novelty detectors the information is finally computed via a neuronal network prediction of damage severity. However, without a detailed knowledge of the underlying fatigue and e.g. fracture properties of the system it will not be possible to extrapolate to failure.

\section{REQUIREMENTS AND CONSEQUENCES OF SELF-REPAIR}

Self-healing is, in a sense, opposite to a degradation process, which as a dissipative process is connected with the production of entropy. In 1877, Boltzmann suggested a definition of entropy using the statistical thermodynamics approach and the concept of microstates as

$$
S=k \ln \Omega
$$

with $k$ as the Boltzmannis constant and $\Omega$ as the number of microstates corresponding to a given macroscopic state of a system. Microstates are the arrangements of energy and matter in the system that are distinguishable at the atomic or molecular level, but are indistinguish- able at the macroscopic level. Any system tends to evolve into a less-ordered and thus more random macrostate that has a larger number of corresponding microstates, and thus the "configurational" entropy S grows.

All processes that lead to degradation (wear, corrosion, fatigue, etc.) often involve interactions with different characteristic length scales. For example, friction and wear involve interactions of microscale and nanoscale asperities and wear particles, capillary interactions, adhesion, chemical molecular bonding. In most cases, these interactions lead to an irreversible energy dissipation and, therefore, to the production of entropy. In many cases, a system can be divided naturally into several scale levels with a limited interaction between hierarchical scales. The entropy production at the macroscale can be therefore compensated by the entropy consumption at another level. Since the entropy is an additive function and the levels of the hierarchy are separated, the net entropy can be presented as the sum of entropies associated with the structures and process at corresponding scale levels as

$$
S_{\text {net }}=S_{\text {macro }}+S_{\text {meso }}+S_{\text {nano }}
$$

where the indices "net", "macro", "meso" and "nano" correspond to the net entropy, macroscale, microscale (mesoscale), and nanoscale (atomic scale) components [128]. For most applications, is the integrity of the macroscale structure (e.g., the absence of cracks, appearance etc.) of predominant interest whereas the mesoscale and nanoscale structure is of lower interest. The integrity of the macroscale structure may be therefore repaired or restored at the expense of the micro- and atomic scale structures.

In the case of the autonomous self-healing using fluid systems as applied while using capsules or capillaries filed with a glue [82-104] excess entropy, $\Delta S_{\text {macro }}$ associated with the macroscale defects, such as cracks or voids is compensated by affecting the mesoscale structure, e.g., by fracture of microcapsules and the release of the fluid, which decreases the degree of order of the microstructure and thus increases the entropy for $\Delta S_{\text {meso }}$. Crack propagation is an irreversible process, because when intermolecular bonds are broken, the energy $\gamma$ is released irreversibly, so the entropy amount $S_{\text {crack }}=\gamma$ $K A / T$ is produced to create a crack with area $A$. The coefficient $0<K<1$ is the fraction of the dissipated energy $Q$ that is consumed for the creation of the crack, whereas the rest of the energy is dissipated. The ideal state without cracks corresponds to the minimum number of microstates and thus to the lowest possible configurational entropy. The crack can be formed in many different ways and the cracked macrostate corresponds to a number of microstates producing excess configurational entropy, $\Delta S_{\text {macro }}$. In a similar manner, when a capsule ruptures and 
its content is released, the configurational entropy grows because mixing occurs. The configurational entropy growth of mixing of two substances is given by

$$
\Delta S_{\text {mixing }}=-R\left(n_{1} \ln X_{1}+n_{2} \ln X_{2}\right)
$$

where $n_{1}$ and $n_{2}$ are the amounts in moles of two pure substances, $X_{1}$ and $X_{2}$ are mole fraction in the solution, and $R$ is the gas constant. Part of this excess entropy can be consumed for healing the bonds at the crack. The net configurational entropy grows, however the growth is not due to the cracking but due to microcapsule rupture and an irreversible decrease of their number.

If $N$ capsules are ruptured to heal the crack with the area $A$, the net entropy production is given by the mesoscale entropy of mixing minus the macroscale entropy of crack healing

$$
\Delta S_{n e t}=\Delta S_{\text {meso }}+\Delta S_{\text {macro }}=N \Delta S_{\text {mixing }}-K \frac{\gamma L}{T}>0
$$

In case $\Delta S_{\text {macro }}<\Delta S_{\text {meso }}$, the healing is done by decreasing the macroscale component of entropy at the expense of the mesoscale component [128]. Crack healing decreases then disorder (and entropy) as observed at the macroscale, while fracture of the microcapsules increases disorder (and entropy) when observed at the mesoscale. Self healing occurs thus autonomically if the net entropy of the system is increasing and if the rate of healing is higher than the rate of degradation; the effectiveness of the healing mechanism can be influenced or perhaps measured using relating microstructure parameters to the entropy.

It is important to stress that self-repair is not simply a material property, but rather a system property. Therefore, it is not realistic to develop universal self-repairing systems being applicable in all sorts of applications. Consequently, it is important to develop new concepts of self-repairing systems with additional values compared to the existing one, which are focused on a well-defined area/field of applications. However, the self-repair mechanisms used in most current systems and devices are not yet as developed as in the complex functional systems as existing in nature.

Existing autonomic self-healing processes need time (rest periods) and energy. However, the end product is neither aesthetically nor functionally perfect. Defects on a nanoscale size are randomly distributed in any material, mechanical loads during the use cause the formation of cracks initiated by the nanoscaled defects, which cause, in time, degradation of the material up to a possible catastrophic failure.

A self-healing mechanism already initiated at a nanoscale offer many advantages for a more effective prevention of further propagation and growth of micro-cracks as has been shown in a recent modelling study [129]. There, the self-healing process of materials with embedded "glue"-carrying cells, in the regime of the onset of the initial fatigue has been studied by three-dimensional numerical simulations within the percolation- model approach. The onset of material fatigue is delayed in such a system by development of a plateaulike time dependence of the material quality. In this low-damage regime, the changes in the conductance and thus in similar transport and response properties of the material can be used as measures of the material quality degradation. A feature found for three dimensions being much more profound than in earlier-studied two-dimensional systems, is the competition between the healing cells. Even for low initial densities of the healing cells, they interfere with each other and reduce each other's effective healing efficiency. Short-range healing means that cells affect their neighbourhood approximately within a distance equal to their size. Thus, in order not to interfere literally, not to waste glue, other healing cells should not be healing this whole neighbourhood; thus they must be about two "shells of influence" away. The exclusion radius is thus at least three times the cell radius, in terms of the center-to-center separation, it is likely even larger, depending on the specific geometry.

Any architecture for self-healing systems should satisfy essential properties like adaptability, dynamicity, awareness, autonomy, robustness and distribution. From this analysis and discussions of the currently active and explored principles of self-repair the following requirements for the design of an ultimate self-healing structure/material can be formulated:

- A reflection mechanism to detect internal or external conditions for which the system should respond to sensing function of damage

- A reasoning mechanism to determine which actions should be taken in order to response to an input from the reflection mechanism-the feedback to repair mechanism, signal transport

- A configuration mechanism to perform necessary changes to repair or optimize the system as directed by reasoning mechanism and activation of the repair mechanism (repair-on-demand) - transport of energy and/or material, repair with the ability to "heal/fill" damage (e.g. volume increase!)

- Repair has to be either attributive or functional, may be single repair (only one time) or repeating repair

- Detection of success and of the status of the structure, recovery of the initial status

- No reduction of the performance of the matrix material by the self-repair functionality; good adhesion and bonding and sufficient thermo-mechanical 
properties of the repair material

- Sufficient high stability of the self-repair functionality during processing and storage

Such systems have been already constructed and will be discussed at two examples:

\subsection{Self-Repairing Strategies and Structures Used in Current Electronic Devices}

One method of self-healing is to design a multiply redundant system that can reconfigure itself when damaged. Re-configurable control systems require three separate functions: failure detection and isolation to determine which components are no longer useful; parameter identification to provide a model of the damaged structure, and online control design that uses information from the other two to re-establish control of the modified structure. This approach has been applied for some time already in re-configurable circuit hardware. This is particularly the case for DRAM. As a typical industry case, the original yield before a built-in self repair (BISR) using redundant elements for repair for a 256-Mbit commodity DRAM in a 0.11 -micron CMOS process is almost 0 , yet the yield of the same product after repair can increase to more than $60 \%$, and even to $80 \%$. Here, the BISR consists of three main blocks: built-in self test (BIST), built-in redundancy analysis (BIRA), and address reconfiguration (AR) [130]. Typically, a BIST circuit consists of a controller, a test pattern generator (TPG), a data comparator, and interface logic. The controller executes the test algorithm and issues commands for the TPG. One of the algorithms used is testing is the Syndrome identification algorithm. This method identifies fail patterns during the test process to increase spare allocation efficiency. After analysis, the AR circuit repairs the memory; e.g. it replaces the faulty memory elements with the fault-free, spare ones. This typically involves address remapping or address decoder reconfiguration. When the memory size increases, the total benefit as a combination of early-market entry benefit, test benefit, development cost and cost for the redundant memory grows quickly, because the yield decreases exponentially when the memory size increases, and thus the BISR design shows its effectiveness in enhancing the yield. Development costs become negligible when production volume is high, because they are constant for the product. Self-repair systems are currently well known and in use in several electronic structures to maintain

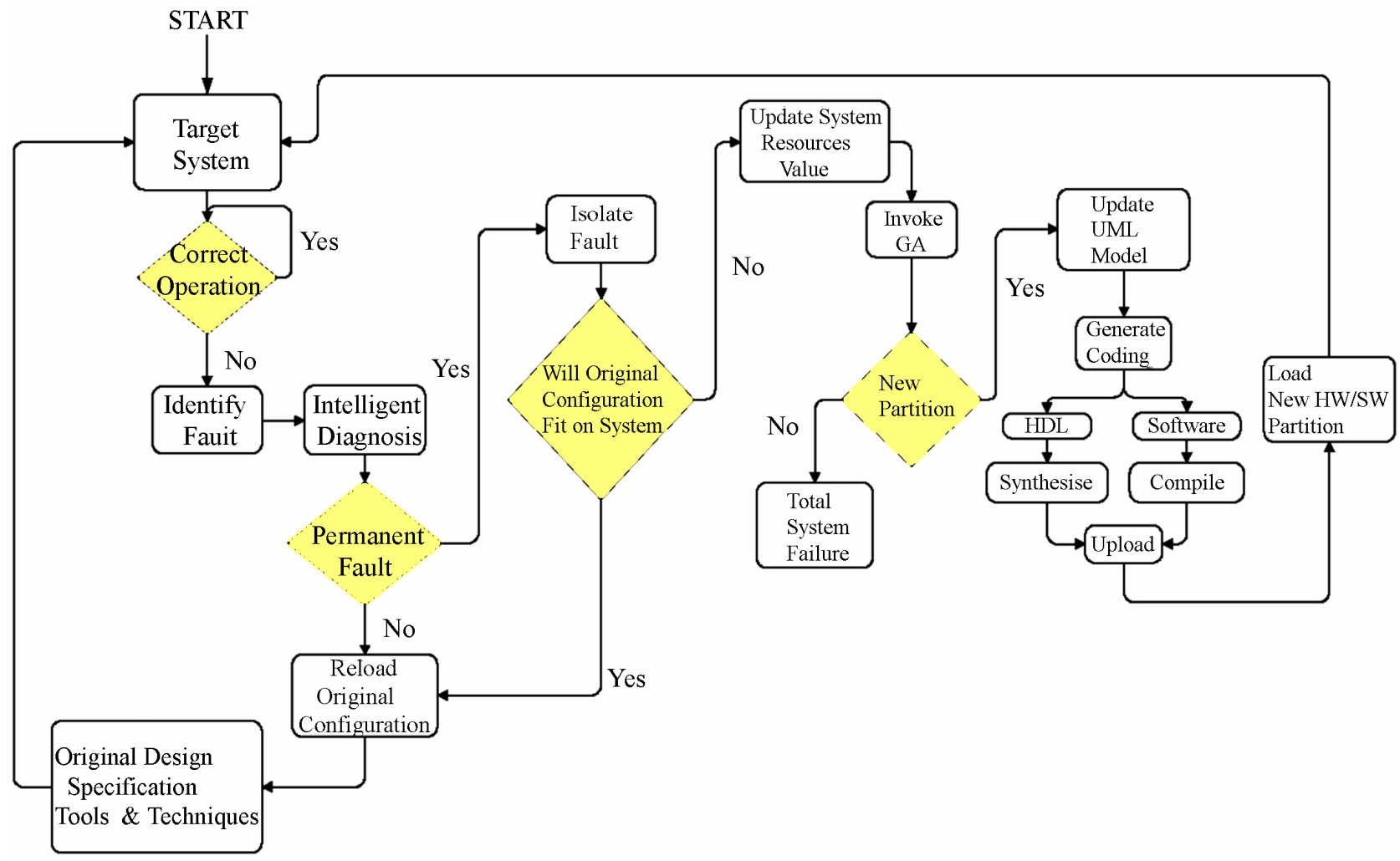

Figure 27. Flow diagram typical for a self-repairing structure used in electronic devices. Reprinted from Engineering Applications of Artificial Intelligence, Self-repair of embedded systems, 17, E. A. Coyle, L. P. Maguire, T. M. McGinnity, 1-9, Copyright (2004), with permission from Elsevier. 
their reliability for a long time. In fact, embedded systems are increasingly entrusted with critical tasks and it becomes crucial that these systems exhibit a high level of reliability. The increasing complexity of such systems makes production of defect-free systems extremely difficult. Therefore, self-repair methodologies were introduced employing a unified modelling language and genetic algorithms and increasing the flexibility and reliability of such systems by enabling the transfer of functionalities between hardware and software. A flow diagram of the processes in such a system is shown in Figure 27.

In contrast to the majority of the already covered systems, this system is an example where the functionality is related to information flow and not to mechanical or aesthetic or other main functionalities. Hence, also the flow of information is used as the signal in the diagnostic process and as the mobile part of the system, able to perform the repair action. The diagnostic circle addresses the functionality during operation of the target system and decides whether the current configuration is able to deal with the isolated fault and to update the system resource value on demand resulting in new partitions, compilation and upload of the new partitions onto the system information. Industrial leaders like Microsoft, Sun Microsystems and IBM are carrying out research on autonomic self-healing systems, grid computing, software agents and middleware computing are typically strategies and software systems and hardware architecture are being developed with self-healing properties. A summary of the developments in this area is given recently by Gosh (2007) [131].

\subsection{Self-Repairing Bolted Joints}

Bolted joint, as one of the most common mechanical components in all types of engineering structures, are critical to the function of the structure and their failure could have huge costs or endanger lives. Unfortunately, bolted joints are subject to a variety of common modes of failure. These include self-loosening, shaking apart, breaking because of corrosion, stress cracking or fatigue, slippage (which can change the way a structure absorbs load, leakage of corrosive substances in the joint), and separation leading to rapid fatigue. One of the most frequent modes of failure for bolted joints is self-loosening. To reduce this mode of failure a concept of a self-sensing and self-healing bolted joint has been developed, consisting of structural members joined together by bolt and nut combinations equipped with piezoceramic and shape memory alloy elements [132] (see Figure 28(a)). This concept combines an impedance-based health- monitoring technique (monitoring of bolt tension and connection damage) together with actuators to restore tension in the system. The actuators are included in the joint as shape memory alloy (SMA) washers. The most common SMA is nickel-titanium, often referred to NiTi or Nitinol, a material that has the ability to convert heat to mechanical energy through a phase transition.

The actuation ability of smart materials should also provide force to a smart structure to counteract damage once it is detected, introducing the possibility of selfhealing structures as demonstrated on this example. The impedance method detects and inspects whether the damage threshold value has been reached or not, and provides a signal to activate the SMA actuator if needed. When damage occurs, temporary adjustments of the bolt tension can be achieved actively and remotely in order to restore lost torque for continued operation (see Figure 28(b)). Thus, this is an example for the construction of a self-monitoring and self-healing system that could be added to existing structures, and provides both condition monitoring and self repair.
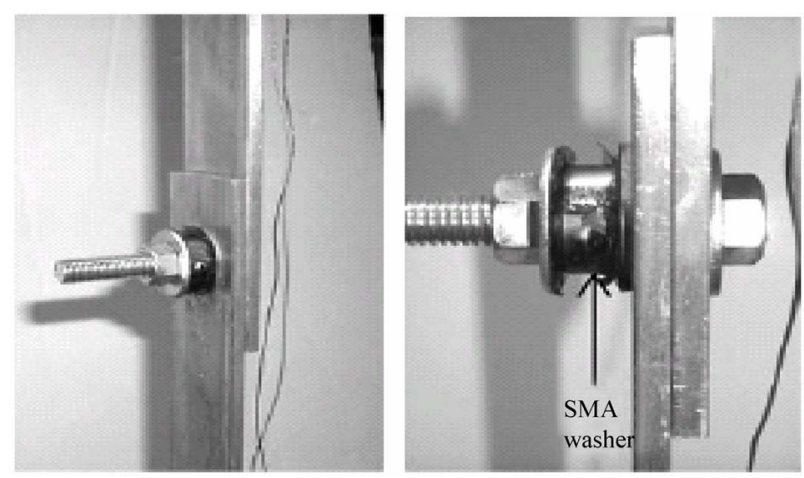

(a)

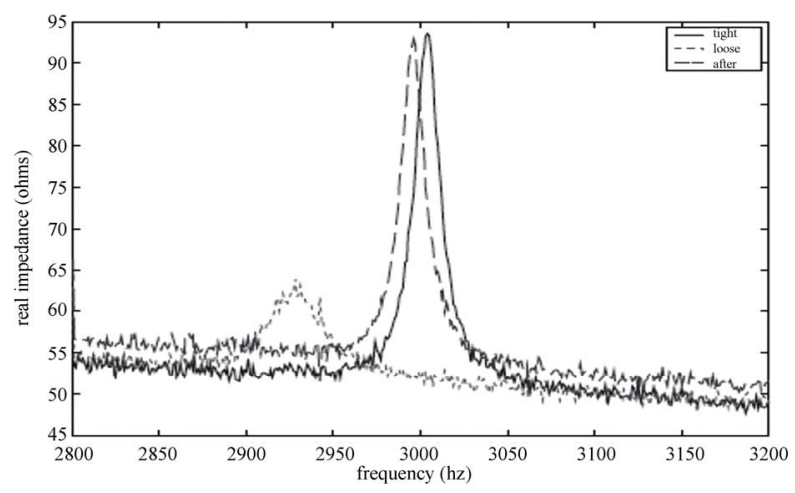

(b)

Figure 28. a) Bolted Joint Configuration with SMA washer and PZT impedance sensor, b) Part of the impedance spectrum of a tight, loose and by SMA actuation fastened bolt connection. Reprinted by permission from IOP Publishing Ltd: [Smart Mater. Struct.] (Practical issues of activating self-repairing bolted joints, D. M. Peairs et al, 13, 1414), copyright (2004). 


\section{TOWARDS A NEW GENERATION OF SELF-HEALING SYSTEMS}

In order to develop a new generation of self-healing devices-smart structures, the following working areas need particular effort:

1) Development/design of sensing elements to be incorporated within structures/surfaces which are able to trigger the self-repair process (signal transport, activation of the repair mechanism). This means that, contrary to most of the model systems, the sensor function has to be further developed and extended with an active learning functionality, able to differentiate and to detect damage, to interpret the obtained information and to trigger/stimulate the repair action on demand. These sensoric elements should be ideally structural parts of the system and should not detoriate the general functionality of the system.

2) Development of transport/repair mechanisms for different activation energies/materials and different circumstances (speed, temperature, amount of recovery). This is especially needed to widen the area of applications of self-healing systems to more materials and systems. Up to now, only a limited number of solutions are existing (encapsulated glues etc.), however, new principles have to be developed and employed to cover a broader range of performance demands.

3) Development of multiple repair processes applicable and sufficient for the purpose. This relates especially to the speed of the repair action, which has to be adjusted to the needs and rest time periods available. Another need is the development of the action-on-demand-only, strongly coupled with the development of sensor/trigger mechanism. If an action-on-demand-only can be realized, then it will be easier to ensure the capability of multiple repair actions. Such an action could be triggered by a concentration of stress which initiates the repair action while activating an initiator. Materials that anticipate damage and increase their strength where damage probability is largest like the system developed by nature for the maintenance of our skeleton are virtually damage free, if enough energy and time for the constant maintenance actions is supplied and given. Alternatively, continuously active materials like present in a living polymerization will realize a perfect restoration of the undamaged state if more reactive monomers are supplied after damage, e.g. via capsules [133].

4) Finally, methods must be developed to test and characterise the structures/devices and to quantify the success of the self-repair action. Up to now, there is only a minor understanding of quantification of the success of self-healing, mostly by measurement of mechanical performance, however, since self-healing is not necessarily only connected to mechanical damage, some measure of the quality of the self-repair has to be developed.

If these requirements are met, then it will be possible to create truly smart structures, which sense their internal state and external environment and based on the information gained respond in a manner that fulfils their functional requirements. The primary advantage of moving towards smart structures technology is the potential cost benefit of condition-based maintenance strategies and the prospective life extension that may be achieved through in-situ health monitoring.

\section{CONCLUDING REMARKS}

Implementation of self-healing is not intended to deal with poor or inadequate application design, development flaws, and problems with the quality of materials or operational errors of systems and devices. However, it should respond to damage caused by external deterioration factors. Thus, self-healing should offer great opportunities for increases in durability and reliability, reduced maintenance and overall costs. This includes reduced material resources, since the usual over-design of materials is no longer required. Repair will be addressed at the very position of first appearance of damage, minimizing the need to have self-repairing functionality throughout the whole system.

Autonomic self-repair should be intrinsically connected with a minimization of the free energy of the system after experiencing damage (e.g. fracture would create additional surfaces and hence enhance the overall energy level of the system). However, this is not as easy and autonomic as it may seem, damage may only lead to another local minimum of the overall free energy and this would be in some cases difficult to detect for a given system. It is for example extremely difficult to detect the energy changes connected with a change in pure appearance of a coating without having scratches and even more difficult to react with a repair action on such changes, however the changes would account for a damage of the esthetical function of the coating. As for now, most of the introduced synthetic self-healing systems have still substantial short-comes, such as the liquid based capsule system, which requires a large amount of capsules to fulfil the function thereby reducing the mechanics of the system in a way that it can only be treated as a model system for fundamental studies [91] or the difficulty in filling and sealing of the liquid based capillary systems which limits applicability [82-90]. Fully autonomous systems need to provide currently a level of mobility which makes them rather unattractive as constructive materials [70]. However, the latest developments show ways for possible solutions such as the application of liquid filled capsules in coating systems or flexible laminates $[134,135]$, here enabling a functional 
repair of a system as corrosion protection which has not the same constraints as a structural material or the use of the same strategy while deploying conductive particles in liquids and as such repairing conductive pathways [136]. The design principles of a self-healing system are universal, however the synthetic system solutions developed so far are mostly special for the very circumstances of the material system and the application (conditions), no universal solution exists applicable for all material systems.

At the most basic level, a self-repairing system requires redundant capacity and the ability to use it effectively. The system must be capable of modifying the configuration of the target system in order to affect a repair. New design principles and strategies will be applied, and finally self-repairing potential will only be incorporated where it is needed or where damage of the material, system or device is going to start. Self-repair will be universal in terms of materials and will follow universal design principles and hence can be applied anywhere. The overall capabilities required for a selfrepairing system extend the application functionality specified by the system designers. The additional elements required include monitoring, test, diagnosis, and repair capabilities. The system monitor must be able to analyse high-level system performance of the system against predefined performance benchmarks, continuously verifying the correctness of the operation of the process and bi-directionally communicating with the target system. The testing process also must have bidirectional communication to activate specific test sequences and receive results. This process should exploit any built-in self-test (BIST) ability, and the testing results are forwarded to the diagnostic process. Automating the fault diagnosis process inherently demands the application of intelligent techniques. Finally, the selfrepair process must be able to affect a repair based on the reduction in resources available, following isolation of the faulty components. If the original level of performance cannot be restored, the monitoring system must be modified to reflect the changes in system characteristics. This feature demands an optimisation capability. Providing these additional capabilities impose a significant burden on the system, there is a trade-off between the additional cost of the system to facilitate self-repair and the potential cost of failure.

The purpose of this reflection is not to provide a review of the knowledge on self-healing systems; therefore, it is by far not covering all work on this subject. The analysis of the systems as discussed in this work shows that the creation of self-healing systems is and is becoming reality. Although a number of synthetically designed self-healing systems are developed and still under development, they mostly fulfil only partially all requirements of an ideal self-healing system as produced by nature.

The knowledge of the system complexity enables the design of a self-healing system for a given application and possible damage. The introduction of self-healing properties into materials systems is with this knowledge and an interdisciplinary approach of design combining all disciplines of science is a growing reality, which has been already demonstrated for a number of synthetic model systems and will have a great future in materials technology especially for systems used in maintenance critical applications such as areas with limited accessibility, high demands of reliability, a guaranteed long life or in areas where repairs cause a lot of hindrance/ annoyance/costs.

\section{AKNOWLEDGEMENTS}

The author acknowledges numerous stimulating discussions within the Delft Centre for Materials (DCMat) and the Innovative Research Program "Self-Healing Materials" of the Dutch Government and especially within the group Fundamentals of Advanced Materials during the weekly stays as Visiting Scientist at the Faculty of Aerospace Engineering at the TU Delft, Netherlands. CSIRO Materials Science and Engineering enabled during a stay as Senior Research Fellow the finalization of the manuscript. Special thanks go to O. Adan TNO/TU Eindhoven and to Tim Harvey, "the butcher", CSIRO for critically reading the manuscript.

\section{REFERENCES}

[1] Fratzl, P. and Weinkamer, R. (2007) Hierarchical structure and repair of bone: Deformation, remodeling, healing. Self Healing Materials, An Alternative Approach to 20 Centuries of Materials Science, Series: Springer Series in Materials Science, 100, 323-335.

[2] Farrar, C.R. and Worden, K. (2007) An introduction to structural health monitoring. Philosophical Transactions of the Royal Society A, 365(1851), 303-315.

[3] Asai, S., Koumoto, K., Matsushita, Y., Yashima, E., Morinaga, M., Takeda, K., Iritani, E., Tagawa, T., Tanahashi, M. and Miyazawa, K.-I. (2003) Advances in nature-guided materials processing. Science and Technology of Advanced Materials, 4(5), 421-433.

[4] Bergman, S.D. and Wudl, F. (2008) Mendable polymers. Journal of Materials Chemistry, 18(1), 41-62.

[5] Wool, R.P. (2008) Self-healing materials: A review. Soft Matter, 4(3), 400-418.

[6] Wu, D.Y., Meure, S. and Solomon, D. (2008) Self-healing polymeric materials: A review of recent developments. Progress in Polymer Science, 33(5), 479-522.

[7] Albert, S.F. (1981) Electrical stimulation of bone repair. Clinical Podiatric Medical Surgery, 8(4), 923-935.

[8] Coyle, E.A., Maguire, L.P. and McGinnity, T.M. (2004) Self repair of embedded systems. Engineering Applications of Artificial Intelligence, 17(1), 1-9. 
[9] Williams, K.A., Dreyer, D.R. and Bielawski, C.W. (2008) The underlying chemistry of self-healing materials. MRS Bulletin, 33(8), 759-765.

[10] de Gennes, P.G. (1971) Reptation of a polymer chain in the presence of fixed obstacles. Journal of Chemical Physics, 55(2), 572-579.

[11] Prager, S. and Tirell, M. (1981) The healing process at polymer-polymer interfaces. Journal of Chemical Physics, 75(10), 5194-5198.

[12] Jud, K. and Kausch, H.H. (1979) Load transfer through chain molecules after interpenetration at interfaces. Polymer Bulletin, 1(1), 697-707.

[13] Wool, R.P. and O'Connor, K.M. (1981) A theory of crack healing in polymers. Journal of Applied Physics, 52(10), 5953-5963.

[14] Kim, Y.H. and Wool, R.P. (1983) A theory of healing at a polymer-polymer interface. Macromolecules, 16(7), $1115-1120$.

[15] Jud, K., Kausch, H.H. and Williams, J.G. (1981) Fracture mechanics studies of crack healing and welding of polymers. Journal of Material Science, 16(1), 204-210.

[16] Boiko, Y. M. and Lyngaae-Jørgensen, J. (2004) Healing of interfaces of high- and ultra-high-molecular-weight polystyrene below the bulk Tg. Polymer, 45(25), 85418549.

[17] Guerin, G., Mauger, F. and Prud'homme, R.E. (2003) The adhesion of amorphous polystyrene surfaces below $\mathrm{Tg}$. Polymer, 44(24), 7477-7784.

[18] Brown, H.R. and Russell, T.P. (1996) Entanglements at polymer interfaces. Macromolecules, 29(2), 798-800.

[19] Silberberg, A. (1988) Distribution of segments near the surface of a melt of linear flexible macromolecules: Effect on surface tension. Journal of Colloid and Interface Science, 125(1), 14-22.

[20] Yamaguchi, M., Ono, S. and Terano, M. (2007) Self-repairing property of polymer network with dangling chains. Materials Letters, 61(6), 1396-1399.

[21] Hayes, S.A., Jones, F.R., Marshiya, K. and Zhang, W. (2007) A self-healing thermosetting composite material. Composites Part A: Applied Science and Manufacturing, 38(4), 1116-1120.

[22] Hayes, S.A., Zhang, W., Branthwaite, M. and Jones, F.R. (2007) Self-healing of damage in fibre-reinforced polymer-matrix composites. Journal of the Royal Society Interface, 4(13), 381-387.

[23] Zako, M. and Takano, N. (1999) Intelligent material systems using epoxy particles to repair microcracks and delamination in GFRP. Journal of Intelligent Material Systems and Structures, 10(10), 836-841.

[24] Meure, S., Wu, D.Y. and Furman, S. (2009) Polyethylene-co-mathacrylic acid healing agents for mendable epoxy resins. Acta Materialia, 57(14), 4312-4320.

[25] Liu, W., Sun, X. and Khaleel, M.A. (2008) Predicting Young's modulus of glass/ceramic sealant for solid oxide fuel cell considering the combined effects of aging, micro-voids and self-healing. Journal of Power Sources, 185(2), 1193-1200.

[26] Seo, S.H., Kim, Y.-W. and Chang, J.Y. (2005) Smectic layered polymer networks based on side chain liquid crystalline polymers having thermally reversible urea bonds. Macromolecules, 38(5), 1525-1527.

[27] Otsuka, H., Aotani, K., Amamoto, Y. and Takahara, A.
(2007) Thermal reorganization and molecular weight control of dynamic covalent polymers containing alkoxyamines in their main chains. Macromolecules, 40(5), 1429-1434.

[28] Mee, M.A.J., Goossens, J.G.P. and v. Duin, M. (2008) Thermoreversible cross-linking of maleated ethylene/ propylene copolymers with diamines and amino-alcohols. Polymer, 49(5), 1239-1248.

[29] Diels, O. and Alder, K. (1928). Synthesen in der hydroaromatischen Reihe. Liebigs Annalen der Chemie, 460(1), 98-122.

[30] Craven, J. M., US patent 3.435.003 (1969).

[31] Chen, X., Dam, M.A., Ono, K., Mal, A., Shen, H., Nutt, S.R., Sheran, K. and Wudl, F., (2002) A thermally re-mendable cross-linked polymeric material. Science, 295(5560), 1698-1702.

[32] Chen, X., Wudl, F., Mal, A., Shen, H. and Nutt, S.R. (2003) New thermally remendable highly cross-linked polymeric materials. Macromolecules, 36(6), 1802-1807.

[33] Murphy, E.B., Bolanos, E., Schaffner-Hamann, C., Wudl, F., Nutt, S.R. and Auad, M.L. (2008) Synthesis and characterization of a single-component thermally remendable polymer network: Staudinger and stille revisited. Macromolecules, 41(14), 5203-5209.

[34] Wouters, M., Craenmehr, E., Tempelaars, K., Fischer, H., Stroeks, N. and van Zanten, J. (2008) Preparation and properties of a novel remendable coating concept. Progress in Organic Coatings, 64(2-3), 156-162.

[35] Schuman T.P. (2007) Smart corrosion inhibition strategies: Substrate, coating, and inhibitors. JCT Coatings Tech, 4(2), 60-70.

[36] Wang, Y., Bolanos, E., Wudl, F., Hahn, T. and Kwok, N. (2007) Self-healing polymers and composites based on thermal activation. Proceedings of SPIE-The International Society for Optical Engineering, 6526, 1-12.

[37] Liu, Y.-L. and Hsieh, C.-Y. (2006) Crosslinked epoxy materials exhibiting thermal remendablility and removability from multifunctional maleimide and furan compounds. Journal of Polymer Science: Part A: Polymer Chemistry, 44(2), 905-913.

[38] Liu, Y.-L. and Chen, Y.-W. (2007) Thermally reversible cross-linked polyamides with high toughness and selfrepairing ability from maleimide- and furan-functionalized aromatic polyamides. Macromolecular Chemistry and Physics, 208(2), 224-232.

[39] Aubert, J.H. (2003) Thermally removable epoxy adhesives incorporating thermally reversible Diels-Alder adducts. The Journal of Adhesion, 79(6), 609-616.

[40] Liu, Y.-L., Hsieh, C.-Y. and Chen, Y.-W. (2006) Thermally reversible cross-linked polyamides and thermoresponsive gels by means of Diels-Alder reaction. Polymer, 47(8), 2581-2586.

[41] Gotsmann, B., Duerig, U., Frommer, J. and Hawker, C.J. (2006) Exploiting chemical switching in a diels-alder polymer for nanoscale probe lithography and data storage. Advanced Functional Materials, 16(11), 1499-1505.

[42] Park, J.S., Takahashi, K., Guo, Z., Wang, Y., Bolanos, E., Hamann-Schaffner, C., Murphy, E., Wudl, F. and Hahn, H.T. (2008) Towards development of a self-healing composite using a mendable polymer and resistive heating. Journal of Composite Materials, 42(26), 2869-2881.

[43] Plaisted, T.A., Amirkhizi, A.V., Arbelaez, D., Nemat- 
Nasser, S.C. and Nemat-Nasser, S. (2003) Self-healing structural composites with electromagnetic functionality. Proceedings of SPIE-The International Society for Optical Engineering, 5054, 372-381.

[44] Kirkby, E.L., Rule, J.D., Michaud, V.J., Sottos, N.R., White, S.R. and Månson, J.-A.E. (2008) Embedded shapememory alloy wires for improved performance of selfhealing polymers. Advanced Functional Materials, 18 (15), 2253-2260.

[45] Li, G. and John, M. (2008) A self-healing smart syntactic foam under multiple impacts. Composites Science and Technology, 68(15-16), 3337-3343.

[46] Brunsveld, L., Folmer, B.J.B., Meijer, E.W. and Sijbesma, R.P. (2001) Supramolecular polymers. Chemical Reviews, 101(12), 4071-4098.

[47] Chino, K. and Ashiura, M. (2001) Thermoreversible cross-linking rubber using supramolecular hydrogenbonding networks. Macromolecules, 34(26), 9201-9204.

[48] Kalista, S.J.Jr. and Ward, T.C. (2007) Thermal characteristics of the self-healing response in poly(ethyleneco-methacrylic acid) copolymers. Journal of the Royal Society Interface, 4(13), 405-411.

[49] Varley, R.J. and v.d. Zwaag, S. (2008) Towards an understanding of thermally activated self-healing of an ionomer system during ballistic penetration. Acta Materialica, 56(19), 5737-5750.

[50] Chung, C.M., Roh, S.Y., Cho, S.Y. and Kim, J.G. (2004) Crack healing in polymeric materials via photochemical [2+2] cycloaddition. Chemistry of Materials, 16(21), 3982-3984.

[51] Scott, T.F., Schneider, A.D., Cook, W.D. and Bowmen, C.N. (2005) Chemistry: Photoinduced plasticity in crosslinked polymers. Science, 308(5728), 1615-1617.

[52] Williams, K.A., Boydston, A.J. and Bielawski, C.W. (2007) Towards electrically conductive, self-healing materials. Journal of the Royal Society Interface, 4(13), 359-362.

[53] Kawagoe, M., Nakanishi, M., Qui, J. and Morita, M. (1997) Growth and healing of a surface crack in poly (methyl methacrylate) under case II diffusion of methanol. Polymer, 38(24), 5969-5975.

[54] Hsieh, H.-C., Yang, T.-J. and Lee, S. (2001) Crack healing in poly(methyl methacrylate) induced by co-solvent of methanol and ethanol. Polymer, 42(3), 1227-1241.

[55] Egloffstein, T.A. (2001) Natural bentonites-Influence of the ion exchange and partial desiccation on permeability and self-healing capacity of bentonites used in GCL. Geotextiles and Geomembranes, 19(7), 427-444.

[56] Shi, C. and Booth, R. (2005) Laboratory Development and field demonstration of self-sealing/self-healing landfill liner. Waste Management, 25(3), 231-238.

[57] Edvardsen, C. (1999) Water permeability and autogenous healing of cracks in concrete. Materials Journal, 96(4), 448-454.

[58] Hearn, N. (1998) Self-sealing, autogenous healing and continued hydration: What is the difference? Materials and Structures/Materiaux et Constructions, 31(8), 563567 ,

[59] Loving, N.W. (1968) Autogenous healing of concrete. American Concrete Pipe Association, Bulletin, 13, 3.

[60] Wagner, E.F. (1974) Autogenous healing of cracks in cement-mortar linings for gray-iron and ductile-iron wa- ter pipe. Journal of American Water Works Association, 66(6), 358-360.

[61] Soroker, V.J. and Denson, A.J. (1926) Autogenous healing of concrete. Zement, 25, 30.

[62] Brandeis, F. (1937) Autogenous healing of concrete. Beton und Eisen, 36, 12.

[63] Powe, T.C., Copeland, L.E., Hayes, J.C. and Mann, H.M. (1954) Permeability of Portland cement pastes. Journal of the American Concrete Institute, 51(3), 285-298.

[64] Sahmaran, M. (2007) Effect of flexure induced transverse crack and self-healing on chloride diffusivity of reinforced mortar. Journal of Materials Science, 42(22), 9131-9136.

[65] Zhong, W. and Yao, W. (2008) Influence of damage degree on self-healing of concrete. Construction and Building Materials, 22(6), 1137-1142.

[66] Li, V.C. and Yang, E.-H. (2007) Self healing in concrete materials. Self Healing Materials, An Alternative Approach to 20 Centuries of Materials Science, Series: Springer Series in Materials Science, 100, 161-193.

[67] Jonker, H. (2007) Self healing in concrete materials. Self Healing Materials, An Alternative Approach to 20 Centuries of Materials Science, Series: Springer Series in Materials Science, 100, 195-204.

[68] Sugama, T. and Gawlik, K. (2003) Self-repairing poly (phenylenesulfide) coatings in hydrothermal environments at $200^{\circ} \mathrm{C}$. Materials Letters, 57(26-27), 4282-4290.

[69] Kim, Y.R., Little, D.N. and Lytton, R.L. (2003) Fatigue and healing characterization of asphalt mixes. Journal of Materials in Civil Engineering (ASCE), 15(1), 75-83.

[70] Cordier, P., Tournilhac, F., Soulié-Ziakovic, C. and Leibler, L. (2008) Self-healing and thermoreversible rubber from supramolecular assembly. Nature, 451(7181), 977-980.

[71] Lumley, R.N., O’Donnell, R.G., Polmear, I.J. and Griffiths, J.R. (2005) Enhanced fatigue resistance by underageing an Al-Cu-Mg-Ag alloy. Matererials Science Forum, 29, 256-261.

[72] Shiya, N., Kyono, J. and Laha, K. (2006) Self-healing effect of boron nitride precipitation on creep cavitation in austenitic stainless steel. Journal of Intelligent Material Systems and Structures, 17(12), 1127-1133.

[73] Abe, O., Ohwa, Y. and Kuranobu, Y.-I. (2006) Possibility of enhanced strength and self-recovery of surface damages of ceramics composites under oxidative conditions. Journal of the European Ceramic Society, 26(4-5), 689-695.

[74] Bennett I.J. and Sloof, W.G. (2006) Modeling the influence of reactive elements on the work of adhesion between a thermally grown oxide and a bond coat alloy. Materials and Corrosion, 57(3), 223-229.

[75] Zhang, X., Xu, L., Du, S., Han, W. and Han, J. (2008) Crack-healing behavior of zirconium diboride composite reinforced with silicon carbide whiskers. Scripta Materialia, 59(11), 1222-1225.

[76] Ando, K., Kim, B.-S., Chu, M.-C., Saito, S., Takahashi, K. (2004) Crack-healing and mechanical behaviour of $\mathrm{A} 12 \mathrm{O} 3 / \mathrm{SiC}$ composites at elevated temperature. Fatique and Fracture of Engineering Materials and Structures, 27(7), 533-541.

[77] Ando, K., Chu, M.-C. and Mastusita, S. (2003) Effect of crack-healing and proof-testing procedures on fatigue 
strength and reliability of Si3N4/SiC composites. Journal of the European Ceramic Society, 23(6), 977-984.

[78] Song, G.M., Pei, Y.T., Sloof, W.G., Li, S.B., De Hosson, J.Th.M. and van der Zwaag, S. (2008) Oxidation-induced crack healing in Ti3AlC2 ceramics. Scripta Materialia, 58(1), 13-16.

[79] Devanathan, R. and Weber, W.J. (2008) Dynamic annealing of defects in irradiated zirconia-based ceramics. Journal of Materials Research, 23(3), 593-595.

[80] Hikasa, A., Sekino, T., Hayashi, Y., Rajagopalan, R. and Niihara, K. (2004) Preparation and corrosion studies of self-healing multi-layered nano coatings of silica and swelling clay. Materials Research Innovations, 8(2), 84-88.

[81] Miccichè, F., Fischer, H., Varley, R. and van der Zwaag, S. (2007) Moisture induced crack filling in barrier coatings containing montmorillonite as an expandable phase. Surface \& Coatings Technology, 202(14), 3346-3353.

[82] Dry, C. (1994) Matrix cracking repair and filling using active and passive modes for smart timed release of chemicals from fibers into cement matrices. Smart Marterials and Structures, 3(2), 118-123.

[83] Dry, C., Dry, C. and McMillan, W. (1996) Three-part methylmethacrylate adhesive system as an internal delivery system for smart responsive concrete. Smart Marterials and Structures, 5(3), 297-300.

[84] Dry, C., (2000) Three designs for the internal release of sealants, adhesives, and waterproofing chemicals into concrete to reduce permeability. Cement and Concrete Research, 30(12), 1969-1977.

[85] Dry, C., Corsaw, M. and Bayer, E. (2003) A comparison of internal self-repair with resin injection in repair of concrete. Journal of Adhesion Science and Technology, 17(1), 79-89.

[86] Dry, C. (1996) Procedure developed for self-repair of polymeric matrix composite materials. Composite Structures, 35(3), 263-269.

[87] Bleay, S.M., Loader, C.B., Hawyes, V.J., Humberstone, L. and Curtis, P.T. (2001) A smart repair system for polymer matrix composites. Composites-Part A: Applied Science and Manufacturing, 32(12), 1767-1776.

[88] Pang, J.W.C. and Bond, I.P. (2005) 'Bleeding composites'-Damage detection and self-repair using a biomimetic approach. Composites Part A: Applied Science and Manufacturing, 36(2), 183-188.

[89] Motuku, M., Vaidya, U.K. and Janowski, G.M. (1999) Parametric studies on self-repairing approaches for resin infused composites subjected to low velocity impact. Smart Materials and Structures, 8(5), 623-638.

[90] Trask, R.S. and Bond, I.P. (2006) Self-healing composite sandwich structures. Smart Materials and Structures, 15(3), 704-710

[91] White, S.R., Sottos, N.R., Geubelle, P.H., Moore, J.S., Kessler, M.R., Sriram, S.R., Brown, E.N. and Viswanathan, S. (2001) Autonomic healing of polymer composites. Nature, 409(6822), 794-779.

[92] Yan, C.Y., Min, Z.R., Ming, Q.Z., Chen, J., Gui, C.Y. and Xue, M.L. (2008) Self-healing polymeric materials using epoxy/mercaptan as the healant. Macromolecules, 41(14), 5197-5202.

[93] Jones, A.S., Rule, J.D., Moore, J.S., Sottos, N.R. and White, S.R. (2007) Self-healing of damage in fi- bre-reinforced polymer-matrix composites. Journal of the Royal Society Interface, 4(13), 395-403.

[94] Cho, S.H., Andersson, H.M., White, S.R., Sottos, N.R. and Brun, P.V. (2006) Polydiniethylsiloxane-based selfhealing materials. Advanced Materials, 18, 997-1000.

[95] Brown, E.N., Sottos, N.R. and White, S.R. (2002) Fracture testing of a self-healing polymer composite. Experimental Mechanics, 42(4), 372-379.

[96] Blaiszik, B.J., Sottos, N.R. and White, S.R. (2008) Nanocapsules for self-healing materials. Composites Science and Technology, 68(3-4), 978-986.

[97] Yin, T., Rong, M.Z., Zhang, M.Q. and Yang, G.C. (2007) Self-healing epoxy composites-preparation and effect of the healant consisting of microencapsulated epoxy and latent curing agent. Composites Science and Technology, 67(2), 201-212.

[98] Kumar, A., Stephenson, L.D. and Murray, J.N. (2006) Self-healing coatings for steel. Progress in Organic Coatings, 55(3), 244-253.

[99] He, X. and Shi, X. (2009) Self-repairing coating for corrosion protection of aluminum alloys. Progress in $\mathrm{Or}$ ganic Coatings, 65(1), 37-43.

[100] Suryanarayana, C., Rao, K.C. and Kumar, D. (2008) Preparation and characterization of microcapsules containing linseed oil and its use in self-healing coatings. Progress in Organic Coatings, 63(1), 72-78.

[101] Sauvant-Moynot, V., Gonzalez, S. and Kittel, J. (2008) Self-healing coatings: An alternative route for anticorrosion protection. Progress in Organic Coatings, 63(3), 307-315.

[102] Caruso, M.M., Blaiszik, B.J., White, S.R., Sottos, N.R. and Moore, J.S. (2008) Full recovery of fracture toughness using a nontoxic solvent-based self-healing system. Advanced Functional Materials, 18(13), 1898-1904.

[103] Caruso, M.M., Delafuente, D.A., Ho, V., Sottos, N.R., Moore, J.S. and White, S.R. (2007) Solvent-promoted self- healing epoxy materials. Macromolecules, 40(25), 8830-8832.

[104] Mookhoek, S.D., Mayo, S.C., Hughes, A.E., Fischer, H.R. and Zwaag, v.d.S. (2010) Applying SEM-based X-ray microtomography to observe self-healing in solvent encapsulated thermoplastic materials. Advanced Engineering Materials, 12(3), 228

[105] Bon, S.A.F., Mookhoek, S.D., Colver, P.J., Fischer, H.R. and van der Zwaag, S. (2007) Route to stable nonspherical emulsion droplets. European Polymer Journal, 43(11), 4839-4842

[106] Carlson, J.A., English, J.M. and Coe, D.J. (2006) A flexible, self-healing sensor skin. Smart Materials and Structures, 15(5), N129-N135.

[107] Toohey, K.S., Sottos, N.R., Lewis, J.A., Moore, J.S. and White, S.R. (2007) Self-healing materials with microvascular networks. Nature Materials, 6(8), 581-585.

[108] Toohey, K.S., Sottos, N.R. and White, S.R. (2009) Characterization of microvascular-based self-healing coatings. Experimental Mechanics, 49(5), 707-717.

[109] Williams, H.R., Trask, R.S., Knights, A.C., Williams, E.R. and Bond, I.P. (2008) Biomimetic reliability strategies for self-healing vascular networks in engineering materials. Journal of the Royal Society Interface, 5(24), 735-747.

[110] Williams, H.R., Trask, R.S., Weaver, P.M. and Bond, I.P. 
(2008) Minimum mass vascular networks in multifunctional materials. Journal of the Royal Society Interface, 5(18), 55-65.

[111] Liu, H.A., Gnade, B.E. and Baalkus, Jr.K.J. (2008) A delivery system for self-healing inorganic films. $A d$ vanced Functional Materials, 18(22), 1-10.

[112] Martin, P. (1997) Wound healing-aiming for a perfect skin regeneration. Science, 276(5309), 75-81.

[113] Lee, J.Y., Buxton, G. and Balazs, A.C. (2004) Using nanoparticles to create self-healing composites. Journal of Chemical Physics, 121(11), 5531-5540.

[114] Tyagi, S., Lee, J.Y., Buxton, G.A. and Balazs, A.C. (2004) Using nanocomposite coatings to heal surfacebdefects. Macromolecules, 37(24), 9160-9168.

[115] Smith, K.A., Tyagi, S. and Balazs, A. (2005) Healing surface defects with polymer nanocomposites containing spheres. AIChE Annual Meeting, Conference Proceedings, 4717.

[116] Smith, K.A., Tyagi, S. and Balazs, A.C. (2005) Healing surface defects with nanoparticle filled polymer coatings: Effect of particle geometry. Macromolecules, 38(24), 10138-10147.

[117] Gupta, S., Zhang, Q., Emrick, T., Balazs, A.C. and Russell, T.P. (2006) Entropy-driven segregation of nanoparticles to cracks in multilayered composite polymer structures. Nature Materials, 5(3), 229-233.

[118] Verberg, R., Dale, A.T., Kumar, P., Alexeev, A. and Balazs, A.C. (2007) Healing substrates with mobile, particle-filled microcapsules: Designing a 'repair and go' system. Journal of the Royal Society Interface, 4(13), 349-357.

[119] Takeda, K., Tanahashi, M. and Unno, H. (2003) Self-repairing mechanism of plastics. Science and Technology of Advanced Materials, 4(5), 435-444.

[120] Shinya, N., Kyono, J. and Laha, K. (2006) Self-healing effect of boron nitride precipitation on creep cavitation in austenitic stainless steel. Journal of Intelligent Material Systems and Structures, 17(12), 1127-1133.

[121] Kessler, S.S. and Spearing, S.M. (2003) Selection of materials and sensors for health monitoring of composite structures. Proceedings of Materials Research Society Symposium, 785, 365-375.

[122] Mal, A., Banerjee, S. and Ricci, F. (2007) An automated damage identification technique based on vibration and wave propagation data. Philosophical Transactions of the Royal Society A, 365(1851), 479-491.

[123] Hayes, S.A. and Hue, L. (2002) A resistance-based damage location sensor for carbon-fibre composites. Smart
Materials and Structures, 11(6), 966-969.

[124] Thostenson, E.T. and Chou, T.-W. (2006) Carbon nanotube networks: Sensing of distributed strain and damage for life prediction and self healing. Advanced Materials, 18(21), 2837-2841.

[125] Thostenson, E.T. and Chou, T.-W. (2008) Carbon nanotube-based health monitoring of mechanically fastened composite joints. Composites Science Technology, 68(12), 2557-2561.

[126] Zhang, W., Sakalkar, V. and Koratkar, N. (2007) In situ health monitoring and repair in composites using carbon nanotube additives. Applied Physics Letters, 91(13), 133102-133104.

[127] Worden, K. and Manson G. (2007) The application of machine learning to structural health monitoring. Philosophical Transactions of the Royal Society A, $\mathbf{3 6 5}$ (1851), 515-537.

[128] Nosonovsky, M., Amano, R., Lucci, J.M. and Rohatgi, P.K. (2009) Physical chemistry of self-organization and self-healing in metals. Physical Chemistry Chemical Physics, 11(41), 9530-9536.

[129] Dementsov, A. and Privman, V. (2008) Three-dimensional percolation modeling of self-healing composites. Physical Review E-Statistical, Nonlinear, and Soft Matter Physics, 78(2), 021104.

[130] Huang, R.-F., Chen, C.-H. and Wu, C.-W. (2007) Economic aspects of memory built-in self-repair. IEEE Design \& Test of Computers, 24(2), 164-172.

[131] Gosh, D., Sharman, R., Rao, H.R. and Upadhaya, S. (2007) Self-healing systems-survey and synthesis. Decision, Support Systems, 42(4), 2164-2185

[132] Peairs, D.M., Park, G. and Inman, D.J. (2004) Practical issues of activating self-repairing bolted joints. Smart Materials and Structures, 13(6), 1414-1423.

[133] Wang, H.-W., Yuan, Y.C., Rong, M.Z. and Zhang, M.Q. (2010) Self-Healing of thermoplastics via living polymerisation. Macromolecules, 43(2), 595-598.

[134] Cho, S.H., White, S.R. and Braun, P.V. (2009) SelfHealing polymer coatings. Advanced Materials, 21(6), 645-649.

[135] Beiermann, B.A., Keller, M.W. and Sottos, N.R. (2009) Self-Healing flexible laminates for resealing of puncture damage. Smart Materials and Structures, 18(8), 1-7.

[136] Caruso, M.M., Schelkopf, S.R., Jackson, A.C., Landry, A.M., Braun, P.V. and Moore, J.S. (2009) Microcapsules containing suspensions of carbon nanotubes. Journal of Materials Chemistry, 19(34), 6093-6096. 